TRANSACTIONS OF THE

AMERICAN MATHEMATICAL SOCIETY

Volume 354, Number 6, Pages $2285-2325$

S 0002-9947(02)02965-3

Article electronically published on February 13, 2002

\title{
A MOMENTUM CONSTRUCTION FOR CIRCLE-INVARIANT KÄHLER METRICS
}

\author{
ANDREW D. HWANG AND MICHAEL A. SINGER
}

\begin{abstract}
Examples of Kähler metrics of constant scalar curvature are relatively scarce. Over the past two decades, several workers in geometry and physics have used symmetry reduction to construct complete Kähler metrics of constant scalar curvature by ODE methods. One fruitful idea- the "Calabi ansatz" - is to begin with an Hermitian line bundle $p:(L, h) \rightarrow\left(M, g_{M}\right)$ over a Kähler manifold, and to search for Kähler forms $\omega=p^{*} \omega_{M}+d d^{c} f(t)$ in some disk subbundle, where $t$ is the logarithm of the norm function and $f$ is a function of one variable.

Our main technical result (Theorem A) is the calculation of the scalar curvature for an arbitrary Kähler metric $g$ arising from the Calabi ansatz. This suggests geometric hypotheses (which we call " $\sigma$-constancy") to impose upon the base metric $g_{M}$ and Hermitian structure $h$ in order that the scalar curvature of $g$ be specified by solving an ODE. We show that $\sigma$-constancy is "necessary and sufficient for the Calabi ansatz to work" in the following sense. Under the assumption of $\sigma$-constancy, the disk bundle admits a one-parameter family of complete Kähler metrics of constant scalar curvature that restrict to $g_{M}$ on the zero section (Theorems B and D); an analogous result holds for the punctured disk bundle (Theorem $\mathrm{C}$ ). A simple criterion determines when such a metric is Einstein. Conversely, in the absence of $\sigma$-constancy the Calabi ansatz yields at most one metric of constant scalar curvature, in either the disk bundle or the punctured disk bundle (Theorem E).

Many of the metrics constructed here seem to be new, including a complete, negative Einstein-Kähler metric on the disk subbundle of a stable vector bundle over a Riemann surface of genus at least two, and a complete, scalar-flat Kähler metric on $\mathbf{C}^{2}$.
\end{abstract}

\section{INTRODUCTION}

The subject of this paper is the explicit construction of complete Kähler metrics with prescribed - usually constant-scalar curvature. The technique, hereafter referred to as the momentum construction, is a combination of two main ideas. The first, which goes back at least to the work of Calabi [4, is that of constructing Kähler forms from Kähler potentials that are essentially functions of one real variable. The prototypical example is the ansatz $\omega=\sqrt{-1} \partial \bar{\partial} f(t)$, where $t=(1 / 2) \log \left(\left|z_{1}\right|^{2}+\cdots+\left|z_{n}\right|^{2}\right)$, the $z_{j}$ are standard complex coordinates on $\mathbf{C}^{n}$, and $f$ is a smooth function of one real variable. As is well-known, if $f(t)=t$, then

Received by the editors May 16, 2001 and, in revised form, October 18, 2001.

1991 Mathematics Subject Classification. Primary 53C55; Secondary 32L05, 53C21, 53C25.

MAS is an EPSRC advanced fellow; ADH was supported in part by JSPS Fellowship \#P-94016 and an NSERC Canada Research Grant. 
$\omega$ descends to the Fubini-Study metric on $\mathbf{P}^{n-1}$, while if $f(t)=e^{t}$, $\omega$ extends to the standard flat Kähler form on $\mathbf{C}^{n}$.

The general setting for a construction of this type is the total space of an Hermitian holomorphic line bundle $p:(L, h) \rightarrow\left(M, \omega_{M}\right)$ over a Kähler manifold. Let $t$ be the logarithm of the fibrewise norm function defined by $h$ and consider the Calabi ansatz

$$
\omega=p^{*} \omega_{M}+2 \sqrt{-1} \partial \bar{\partial} f(t)
$$

which associates a Kähler form to (suitably convex) functions of one real variable.

The second idea comes from symplectic geometry and involves a change of variables $f(t)$ to $\varphi(\tau)$ closely related to the Legendre transform. For each $f$, (1.1) is invariant under the circle-action that rotates the fibres of $L$. Let $X$ be the generator of this action, normalized so that $\exp (2 \pi X)=1$. Denote by $\tau$ the corresponding moment map determined (up to an additive constant) by

$$
i_{X} \omega=-d \tau
$$

(In fact $\tau=f^{\prime}(t)$, see Section 2.1.) When $\omega$ comes from the Calabi ansatz, the function $\|X\|_{\omega}^{2}$ is constant on the level sets of $\tau$, so there is a function $\varphi: I \rightarrow$ $(0, \infty)$ - to be called the momentum profile of $\omega$ - such that

$$
\varphi(\tau)=\|X\|_{\omega}^{2}
$$

The crucial point is that $\omega$ can be reconstructed explicitly from its profile; indeed $t$ and $\tau$ are related by the Legendre transform, and the Legendre dual $F$ of $f$ satisfies $F^{\prime \prime}=1 / \varphi$.

The description of $\omega$ in terms of $\varphi$ has many advantages. At one level, this is to be expected: (1.3) shows that $\varphi$ is a canonical geometric quantity, while $f$ determines $\omega$ only through its second derivative. In particular, the only condition that is needed for $\varphi$ to determine a Kähler metric is that it be positive on (the interior of) $I$, see (1.3). Positivity of (1.1), by contrast, corresponds to two conditions on the derivatives of $f$. Further, the geometry of the metric - for example its completeness or extendability properties near the fixed-point set of the $S^{1}$-action-are easily read off from the behaviour of $\varphi$ near the endpoints of $I$. However, the decisive and most remarkable advantage, as far as the problem of prescribed scalar curvature is concerned, is that the scalar curvature of $\omega$ is given by a linear second-order differential expression in $\varphi(\tau)$, in contrast to the fully nonlinear fourth-order function that arises in the $f(t)$-description. Consequently, the profiles that give rise to metrics of constant scalar curvature are explicit rational functions of $\tau$.

This paper is organized as follows. The remainder of the introduction summarizes our main results. Section 2 gives a self-contained account of the momentum construction and Section 3 applies the method to give general existence theorems for complete Kähler metrics of constant scalar curvature. Section 4 is devoted mostly to a discussion of the scope and limitations of the momentum construction, and to examples of bundles to which the results of Section 3 apply. We also describe some possible extensions of this work to metrics of finite fibre area, and give brief, comparative accounts of prior work on the Calabi ansatz. 
Acknowledgements. It is a pleasure to acknowledge the authors whose work laid the foundations for the present article. The most important sources of inspiration were the papers of Calabi [4, Koiso-Sakane [16], LeBrun [19], and PedersenPoon [27]. Related work of Koiso [15], Mabuchi [22], and Simanca [30] also provided guidance and insights.

The first-named author was supported in part by a JSPS Postdoctoral Research Fellowship during the early stages of this work, and by an NSERC Canada Individual Research Fellowship. He is especially grateful to: Professors Y. Sakane and T. Mabuchi, and to Osaka University, for kind and extensive hospitality; the Mathematics Institute at the University of Edinburgh for hospitality and support during the early stages of this work; and to Professor J. Bland, of the University of Toronto, for many patient and helpful discussions. The second-named author is an EPSRC advanced fellow. He is also grateful to the University of Toronto for its hospitality while some of this work was being carried out.

Results about the momentum construction. In the rest of the paper, $p$ : $(L, h) \rightarrow\left(M, \omega_{M}\right)$ is an Hermitian holomorphic line bundle, with curvature form $\gamma=-\sqrt{-1} \partial \bar{\partial} \log h$, over a Kähler manifold of complex dimension $m$. A compatible momentum interval is an interval $I \subset \mathbf{R}$, such that the closed $(1,1)$-form $\omega_{M}(\tau):=$ $\omega_{M}-\tau \gamma$ is positive for every $\tau \in I$. The associated Kähler metric is denoted $g_{M}(\tau)$.

Definition 1.1. Horizontal data $\left\{p:(L, h) \rightarrow\left(M, g_{M}\right), I\right\}$ consists of an Hermitian holomorphic line bundle over a Kähler manifold, together with a compatible momentum interval. The whole assemblage is often denoted $\{p, I\}$ for brevity. A momentum profile is a function $\varphi$ that is smooth on the closure of $I$ and positive on the interior of $I$.

The completion of a line bundle $L$ is the $\mathbf{P}^{1}$ bundle $\widehat{L}=\mathbf{P}(\mathcal{O} \oplus L)$, containing $L$ as a Zariski-open subset and obtained by adding a copy of $M$ 'at $\infty$.' Let $r: \widehat{L} \rightarrow[0, \infty]$ denote the continuous extension of the square of the Hermitian norm function. All the metrics constructed in this paper live on subsets of $\widehat{L}$ obtained by restricting $r$ to an interval (or on manifolds obtained by partially collapsing the zero and/or infinity sections).

Definition 1.2. Let $\mathrm{J} \subset[0, \infty]$ be an open interval. The corresponding invariant subbundle $L^{\prime} \subset \widehat{L}$ is the $S^{1}$-invariant domain $r^{-1}(\mathrm{~J})$.

Different choices of $\mathrm{J}$ yield six distinct complex-analytic fibre types: $\mathrm{J}=[0, \infty]$ (the projective line); $\mathrm{J}=[0, \infty)$ (the complex line); $\mathrm{J}=(0, \infty)$ (the punctured line); $\mathrm{J}=[0,1)$ (the disk); $\mathrm{J}=(0,1)$ (the punctured disk); and $\mathrm{J}=\left(e^{-l}, e^{l}\right)$ (annuli). In the last three cases, homotheties have been used to reduce $\mathrm{J}$ to a standard form. The invariant subbundles corresponding to the first five cases will be denoted $\widehat{L}, L$, $L^{\times}, \Delta(L)$ and $\Delta^{\times}(L)$ respectively. Annulus-subbundles will not play a major role in what follows.

Definition 1.3. Let $L^{\prime} \subset \widehat{L}$ be an invariant subbundle. A bundle-adapted metric on $L^{\prime}$ is a Kähler metric $g$ whose Kähler form $\omega$ arises from the Calabi ansatz (1.1).

The heart of the momentum construction is the fact, implicitly due to Calabi and Koiso-Sakane, that if horizontal data are given, then each momentum profile determines a unique isometry class of bundle-adapted Kähler metric enjoying the geometric properties of equations (1.1)-(1.3): 
Proposition 1.4. Let horizontal data $\{p, I\}$ and a momentum profile $\varphi$ be given. Then there exists an invariant subbundle $L^{\prime} \subset \widehat{L}$, unique up to homothety, and a bundle-adapted Kähler metric $g_{\varphi}$ on $L^{\prime}$, unique up to isometry, with the following properties:

(i): The Kähler form $\omega_{\varphi}$ of $g_{\varphi}$ arises from the Calabi ansatz;

(ii): The image of the moment map $\tau$ is $I$;

(iii): If $X$ generates the circle action, and is normalized so that $\exp (2 \pi X)=\mathrm{Id}$, then $g_{\varphi}(X, X)=\varphi(\tau)$.

It is natural to ask how the geometric properties, especially completeness and the scalar curvature $\sigma_{\varphi}$ of $g_{\varphi}$, are encoded by $\varphi$. As will be seen in Section 2.2 below, completeness of $g_{\varphi}$ is encoded by the boundary behaviour of $\varphi$, in fact by the 2-jet at the endpoints of $I$. Our main technical result is that in momentum coordinates, the scalar curvature is linear in $\varphi$ :

Theorem A. Let horizontal data be given. For each $\tau \in I$, let $\sigma_{M}(\tau)$ denote the scalar curvature of $g_{M}(\tau)$, and define $Q: I \times M \rightarrow \mathbf{R}$ by $Q(\tau)=\omega_{M}(\tau)^{m} / \omega_{M}^{m}$. Then the scalar curvature of $g_{\varphi}$ is given by

$$
\sigma_{\varphi}=\sigma_{M}(\tau)-\frac{1}{2 Q} \frac{\partial^{2}}{\partial \tau^{2}}(Q \varphi)(\tau) .
$$

To interpret (1.4), regard the $S^{1}$-invariant function $\sigma_{\varphi}$ on $L^{\prime}$ as a function on $I \times M$ by factoring through the $S^{1}$-action.

The improvement of Theorem $\mathrm{A}$ over earlier results is that no curvature hypotheses are imposed on the horizontal data. Of course, it is too much to expect that for arbitrary horizontal data, $\sigma_{\varphi}$ can be made constant by an appropriate choice of profile (a single function of one variable). Natural sufficient curvature hypotheses are to assume the two terms in (1.4) separately depend only on $\tau$ for every profile:

Definition 1.5. Horizontal data are said to be $\sigma$-constant if

(i): The curvature endomorphism $\mathrm{B}=\omega_{M}^{-1} \gamma$ has constant eigenvalues on $M$;

(ii): The metric $g_{M}(\tau)$ has constant scalar curvature for each $\tau \in I$.

The simplest examples of $\sigma$-constant horizontal data are pluricanonical bundles over Einstein-Kähler manifolds. More generally, if $g_{M}$ has constant scalar curvature, and $\gamma(L, h)$ is a multiple of $\omega_{M}$, then the data $p:(L, h) \rightarrow\left(M, \omega_{M}\right)$ are $\sigma$-constant. More specific examples appear in Section 4

The conclusion of Theorem $\mathrm{A}$ ] is due to Guan [10] and Hwang [12] under stronger curvature hypotheses (" $\rho$-constancy," see Section 4.5). The corresponding statement for $\sigma$-constant data is used repeatedly in the sequel:

Corollary A.1. Assume the horizontal data $\{p: L \rightarrow M, I\}$ are $\sigma$-constant. Then $\sigma_{M}(\tau)=P / 2 Q$, where $Q$ is as in Theorem $\square$, $P$ is a polynomial in $\tau$, and for each momentum profile $\varphi$,

$$
\sigma_{\varphi}=\frac{1}{2 Q}\left(P-(Q \varphi)^{\prime \prime}\right)(\tau) .
$$

Metrics of constant scalar curvature on line bundles. The next two theorems demonstrate "sufficiency" of $\sigma$-constancy in the Calabi ansatz, for the disk bundle and punctured disk bundle respectively. In each statement, we write " $\gamma \prec 0$ " to indicate that the curvature form $\gamma$ is non-positive, but not identically zero, see 
Remark 1.6 below. The resulting metrics have fibres of infinite area; systematic treatment of metrics of finite fibre area is outside the scope of this paper, though some aspects are discussed in Section 4

Theorem B. Let $I=[0, \infty)$, and let $\{p, I\}$ be $\sigma$-constant horizontal data with $\gamma \prec 0$, and with $g_{M}$ complete. Then there exists a real number $c_{0}$ with the following property: For every $c<c_{0}$, and for at most finitely many $c>c_{0}$, the disk bundle $\Delta(L)$ carries a complete Kähler metric $g_{c}$, of scalar curvature $c$, whose restriction to the zero section is $g_{M}$. When $c=c_{0}$, the analogous conclusion holds, but the metric lives on the total space of $L$. For $c \leq 0$, the metric $g_{c}$ is Einstein if and only if $\frac{c}{m+1} \omega_{M}=\rho_{M}+\gamma$.

An analogue of Theorem $\mathbb{B}$ holds for punctured disk bundles. Of course, the metrics are not obtained from the metrics in Theorem $B$ by restriction, since removing the zero section leaves an incomplete metric.

Theorem C. Let $I=(0, \infty)$, and let $\{p, I\}$ be $\sigma$-constant horizontal data with $\gamma \prec 0$, and with $g_{M}$ complete. Then there exists a real number $c_{0}^{\times}$with the following property: For every $c<c_{0}^{\times}$, and for at most finitely many $c>c_{0}^{\times}, \Delta^{\times}(L)$ carries a complete Kähler metric $g_{c}^{\times}$, of scalar curvature $c$, whose symplectic reduction at $\tau=1$ is $g_{M}-\gamma$. When $c=c_{0}^{\times}$, the analogous conclusion holds, but the metric lives on the total space of $L^{\times}$. For $c<0$, the metric $g_{c}$ is Einstein if and only if $\frac{c}{m+1} \omega_{M}=\rho_{M}$.

Remark 1.6. The area form of the fibre pushes forward under $\tau$ to $2 \pi$ times Lebesgue measure on $I$; thus an end of the fibre has infinite area if and only if the corresponding end of $I$ is unbounded. When the fibre area is infinite, the momentum interval $I$ restricts the curvature form $\gamma$ (since the "horizontal form" $\omega_{M}(\tau)=\omega_{M}-\tau \gamma$ is required to be positive for all $\left.\tau \in I\right)$. In particular, the condition $(0, \infty) \subset I$ implies the curvature $\gamma$ is non-positive. There are dual statements with $\gamma \geq 0$ and $(-\infty, 0) \subset I$, but these yield no new isometry classes of metric, see Lemma 4.1 below (where the assumption inf $I=0$ is also justified). We exclude the case of a flat line bundle $(\gamma=0)$ since this leads only to local product metrics.

Remark 1.7. The constants $c_{0}$ and $c_{0}^{\times}$are roots of polynomials in one variable and can be estimated in terms of the horizontal data. In good cases they can be found exactly. In particular, if $\gamma$ is negative-definite then $c_{0}=0$ and one has the pleasant conclusion that for every $c<0, \Delta(L)$ admits a complete Kähler metric with scalar curvature $c$, while $L$ admits a complete scalar-flat Kähler metric.

Theorem B contains many previously-known results (see Section 4), but even when $M$ is a complex curve, an interesting new result is obtained:

Corollary B.1. Let $M=\mathbf{C}, \omega_{M}=\frac{\sqrt{-1}}{2} d z \wedge d \bar{z}$ the standard flat Kähler form, $(L, h) \rightarrow M$ the trivial line bundle equipped with an Hermitian metric of curvature $\gamma=-2 \pi k \omega_{M}$, ( $k$ a positive constant). Then the total space of $L$-biholomorphic to $\mathbf{C}^{2}$ - admits a complete, scalar-flat Kähler metric that is not Ricci-flat, and whose fibre metric is asymptotically cylindrical.

The importance of this example is that scalar-flat Kähler metrics on complex surfaces are anti-self-dual (in the sense of 4-dimensional conformal geometry), and are thus of independent interest. 
The following Einstein-Kähler metrics arising from Theorem B are due to Calabi [4] when the base is Einstein-Kähler (that is, when $n=1$ in Corollary B.2 below). Moreover, non-homothetic metrics are obtained in Corollary B.2 by scaling the $\omega_{M_{i}}$ separately. In Corollary B.3. further metrics arise by tensoring with a flat line bundle when the base is not simply-connected.

Corollary B.2. For $1 \leq i \leq n$, let $p_{i}: K_{i} \rightarrow M_{i}$ be the canonical bundle of a compact Einstein-Kähler manifold of positive curvature. Then there is a complete, Ricci-flat Kähler metric on the total space of $\bigotimes_{i} p_{i}^{*} K_{i}$.

Corollary B.3. For $1 \leq i \leq n$, let $p_{i}: K_{i} \rightarrow M_{i}$ be a compact Einstein-Kähler manifold of positive curvature. Then for all $\ell>1$, there is a complete EinsteinKähler metric of negative curvature on the disk subbundle of $\left(\bigotimes_{i} p_{i}^{*} K_{i}\right)^{\ell}$. If $M_{i}$ are complete, negative Einstein-Kähler manifolds, then the disk subbundle in $\left(\bigotimes_{i} p_{i}^{*} K_{i}^{-1}\right)^{\ell}$ admits a complete, negative Einstein-Kähler metric.

Theorem C yields some new Einstein-Kähler metrics on punctured disk bundles. The fibre metric has one end of finite area and one end of infinite area.

Corollary C.1. Let $p: L \rightarrow\left(M, g_{M}\right)$ be a holomorphic line bundle over a complete, negative Einstein-Kähler manifold, and assume $c_{1}(L) \in H^{1,1}(M, \mathbf{Z})$ is represented by a form $\gamma$ whose eigenvalues with respect to $\omega_{M}$ are constant on $M$. Then $\Delta^{\times}(L)$ admits a complete, negative Einstein-Kähler metric.

Metrics of constant scalar curvature on vector bundles. The Calabi ansatz applies, in modified form, to vector bundles $E \rightarrow D$ of rank $n>1$; Calabi's construction of a complete, Ricci-flat metric on the cotangent bundle of a compact, rank-one Hermitian symmetric space 4] is the prototypical example. In seeking generalizations of this example, one adopts the following strategy. Take $M=\mathbf{P}(E)$, $L=\tau_{E}$ the tautological line bundle over $\mathbf{P}(E)$. Since the total space of $L$ is the blow-up along the zero-section of $E$, a metric on $E$ is the same thing as a suitably degenerate metric on $\tau_{E}$. The momentum construction is easily modified to produce such partially degenerate metrics on $L$ :

Theorem D. Let $(E, h) \rightarrow\left(D, g_{D}\right)$ be an Hermitian holomorphic vector bundle of rank $n$ over a complete Kähler manifold of dimension $d$, and let $p:\left(\tau_{E}, h\right) \rightarrow$ $M=\mathbf{P}(E)$ be the tautological bundle with the induced Hermitian structure. Let $\gamma$ denote the curvature form of $\left(\tau_{E}, h\right)$. Assume $\omega_{M}(\tau):=\omega_{D}-\tau \gamma$ is a Kähler form on $M$ for all $\tau>0$, and that $p:\left(\tau_{E}, h\right) \rightarrow\left(M, g_{M}\left(\tau_{0}\right)\right)$ is $\sigma$-constant for some $\tau_{0}>0$. Then there is a $c_{0}$ such that for every $c<c_{0}$, and for at most finitely many $c>c_{0}$, the momentum construction determines a complete Kähler metric with scalar curvature $c$ on the disk subbundle $\Delta \subset E$. When $c \leq 0$, this metric is Einstein if and only if $\frac{c}{n+d} \omega_{D}=\rho_{M}+n \gamma$. Furthermore, there is a complete Kähler metric on $E$ with scalar curvature $c_{0}$.

Unfortunately, $\sigma$-constancy of the data $\tau_{E} \rightarrow \mathbf{P}(E)$ is a strong assumption. It is not even sufficient for $E$ to be a homogeneous vector bundle, as the example $E=\mathcal{O} \oplus \mathcal{O}(k) \rightarrow \mathbf{P}^{1}(k<0)$ shows. In this case $\mathbf{P}(E)$ is a Hirzebruch surface, which does not admit a Kähler metric of constant scalar curvature, so condition (ii) in the definition of $\sigma$-constancy is never satisfied.

Each of the next three corollaries contains tractable hypotheses that guarantee the applicability of Theorem D. In Corollary D.1, it is assumed that both $E$ and 
$\mathbf{P}(E)$ are homogeneous. The pseudoconvexity hypothesis guarantees a metric of non-positive curvature on the tautological bundle. The hypotheses of Corollary D.2 imply that $\mathbf{P}(E)$ is a product and that $\tau_{E}$ is a tensor product of line bundles pulled back from the factors. Corollary D.3 which rests on the result of NarasimhanSeshadri to the effect that a stable bundle over a curve is projectively flat, gives families of examples depending on continuous parameters in the event that the base of $E$ is one-dimensional. As above, the restriction of the advertised metric to the zero section is the base metric $g_{D}$. In particular, varying the scalar curvature does not vary the metric through homotheties.

Corollary D.1. Let $E \rightarrow D$ be a homogeneous vector bundle of rank $n>1$ over a compact, homogeneous Kählerian manifold. Assume further that the projective bundle $M=\mathbf{P}(E)$ is homogeneous, and that the zero section of $E$ has a pseudoconvex tubular neighborhood. Endow the base with a homogeneous Kähler metric $g_{D}$, and the bundle with a homogeneous Hermitian metric $h$. Then for every $c<0$, there is a complete Kähler metric $g_{c}$ of scalar curvature $c$ on the disk bundle $\Delta$, and there is a complete, scalar-flat metric on the total space of $E$.

Corollary D.2. Let $\left(D, g_{D}\right)$ be complete, and suppose that $(\Lambda, h) \rightarrow\left(D, g_{D}\right)$ is $\sigma$-constant, with curvature $\gamma<0$. Then the total space of $E=\Lambda \otimes \mathbf{C}^{n}=\Lambda^{\oplus n}$ admits a complete, scalar-flat metric, and for every $c<0$, the disk bundle $\Delta$ (taken with respect to the natural Hermitian structure) admits a complete Kähler metric of scalar curvature $c$.

Corollary D.3. Let $p: E \rightarrow C$ be a stable vector bundle of rank $n$ and degree $k \leq 0$ over a compact Riemann surface $C$, of genus $g \geq 2$ and endowed with the unitarea metric of constant Gaussian curvature. Then the total space of E admits a complete, scalar-flat Kähler metric, and there exists an Hermitian structure $h$ such that for every $c<0$, the disk subbundle of $(E, h)$ carries a complete Kähler metric of scalar curvature $c$. Finally, the metric in this family that has scalar curvature $c=2 \pi(n+1)(2-2 g+k)<0$ is Einstein-Kähler.

Limitations of the Calabi ansatz. If horizontal data are $\sigma$-constant, then by (i) of Definition 1.5, $Q(\tau)$ is constant for all $\tau \in I$, so the second term in (1.4) depends only on $\tau$ for every profile $\varphi$, while (ii) says exactly that $\sigma_{M}(\tau)$ is constant for all $\tau$. Conversely, it is not difficult to show that if, for every $\varphi, \sigma_{\varphi}$ depends only upon $\tau$ then the horizontal data are $\sigma$-constant. We believe the same conclusion follows merely if there exists a profile inducing a metric of constant scalar curvature, but are at present able to prove only a partial result in this direction:

Theorem E. Let $\{p, I\}$ be horizontal data with $I=[0, \varepsilon)$ for some $\varepsilon>0$, and assume there exist real-analytic functions $\sigma_{1}$ and $\sigma_{2}$ on $I$, and distinct profiles $\varphi_{1}$ and $\varphi_{2}$, with $\varphi_{1}(0)=\varphi_{2}(0)$ and inducing metrics of scalar curvature $\sigma_{1}(\tau)$ and $\sigma_{2}(\tau)$, respectively. Then the horizontal data are $\sigma$-constant.

Theorem $\mathrm{E}$ is proved by considering the difference $\varphi_{1}-\varphi_{2}$, and is of course purely local. A simple but suggestive corollary is that on horizontal data that are not $\sigma$-constant, there is at most one metric of constant scalar curvature arising from the Calabi ansatz. In the statement below, it is not necessary to assume $c_{1} \neq c_{2}$; if $c_{1}=c_{2}$, then $\varphi_{1}$ and $\varphi_{2}$ are distinct if and only if $\varphi_{1}^{\prime}(0) \neq \varphi_{2}^{\prime}(0)$. 
Corollary E.1. Let $\{p, I\}$ be horizontal data as above, and assume there exist distinct profiles $\varphi_{1}$ and $\varphi_{2}$, equal at 0 and inducing metrics of scalar curvature $c_{1}$ and $c_{2}$, respectively. Then the horizontal data are $\sigma$-constant.

There does not seem to be an easy way to prove $\sigma$-constancy by assuming existence of one profile, without additional hypotheses. Further partial results and a conjectural strengthening of Theorem E are described in Section 4.

\section{Bundle-Adapted Metrics}

This section sketches the main points in the momentum construction. The details are straightforward calculations; a few simple but helpful observations - as well as information on notational conventions - are included for readers who wish to apply this machinery in other situations.

2.1. The Momentum Construction. Terminology is as in Definitions 1.1 1.3 The following notational conventions are used systematically: A Kähler metric is denoted $g$. Its Kähler form, Ricci form, scalar curvature, and Laplace operator (acting on functions) are denoted $\omega, \rho, \sigma$, and $\square$ respectively. Subscripts are used descriptively when several metrics are under consideration.

Fix $b \leq \infty$. Let $I \subset \mathbf{R}$ be an interval with interior $(0, b)$, and pick $\tau_{0} \in(0, b)$. For each profile $\varphi$, define

$$
t_{1}=\lim _{\tau \rightarrow 0^{+}} \int_{\tau_{0}}^{\tau} \frac{d x}{\varphi(x)}, \quad t_{2}=\lim _{\tau \rightarrow b^{-}} \int_{\tau_{0}}^{\tau} \frac{d x}{\varphi(x)},
$$

noting that $-\infty \leq t_{1}<t_{2} \leq \infty$. Introduce functions $\mu, s$, and $f$ of $t$ by

$$
t=\int_{\tau_{0}}^{\mu(t)} \frac{d x}{\varphi(x)}, \quad s(t)=\int_{\tau_{0}}^{\mu(t)} \frac{d x}{\sqrt{\varphi(x)}}, \quad f(t)=\int_{\tau_{0}}^{\mu(t)} \frac{x d x}{\varphi(x)} .
$$

Then the equations

$$
\mu^{\prime}=\varphi \circ \mu, \quad s^{\prime}=\sqrt{\varphi \circ \mu}, \quad f^{\prime}=\mu
$$

hold on $\left(t_{1}, t_{2}\right)$.

Suppose now that $\left\{p:(L, h) \rightarrow\left(M, g_{M}\right), I\right\}$ are horizontal data, and let $t: L^{\times} \rightarrow$ $\mathbf{R}$ be the logarithm of the norm function. The functions $\mu, s$, and $f$ of $(2.2)$ induce respective functions $\tau:=\mu(t), s(t)$, and $f(t)$ on $L^{\prime}=\left\{z \in L^{\times}: t_{1}<t(z)<t_{2}\right\}$, and determine a closed $(1,1)$-form

$$
\begin{aligned}
\omega_{\varphi} & =p^{*} \omega_{M}+d d^{c} f(t) \\
& =p^{*} \omega_{M}+2 \sqrt{-1} \partial \bar{\partial} f(t) .
\end{aligned}
$$

It transpires that the closed $(1,1)$-form $\omega_{\varphi}$ is positive. The associated bundleadapted metric is denoted $g_{\varphi}$, and the mapping $\varphi \longmapsto \omega_{\varphi}$ is called the momentum construction.

The fibre metric. If $z^{0}$ is a linear coordinate on a fibre of $L$ then the restriction of $g_{\varphi}$ to the fibre is given by

$$
g_{\text {fibre }}=\varphi(\tau)\left|\frac{d z^{0}}{z^{0}}\right|^{2} .
$$

Thus $\varphi(\tau)$ is interpreted as the conformal factor relating $g_{\text {fibre }}$ to the flat metric on the cylinder. The function $s$ of (2.2) is the geodesic distance for $g_{\text {fibre }}$, while $2 \pi(\mu(b)-\mu(a))$ is the area of the subset of the fibre given by $a \leq t \leq b$. 


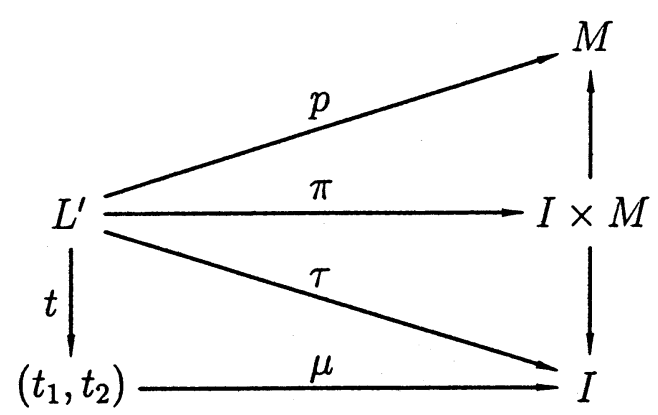

FigurE 2.1. Relation between maps in the momentum construction

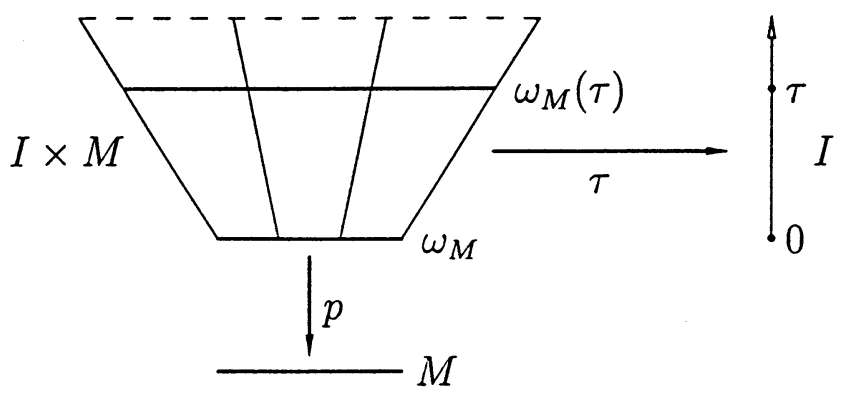

Figure 2.2. Symplectic reduction of $g_{\varphi}$

$S^{1}$-invariant functions on $L$. Fix a bundle-adapted metric on $L^{\prime}$. The functions $t$ and $\tau=\mu(t)$ have the same level sets on $L^{\prime}$, but while $t$ depends only on the Hermitian structure of $L, \tau$ depends on the profile as well. By a customary abuse of notation, $t$ and $\tau$ are regarded as variables in the intervals $\left(t_{1}, t_{2}\right)$ and $I$, respectively.

Define $\pi: L^{\prime} \rightarrow I \times M$ by $\pi=(\tau, p)$. Each fibre of $\pi$ is an orbit of the $S^{1}$-action, so circle-invariant tensors on $L^{\prime}$ may be identified with tensors on $I \times M$; this will henceforth be done freely, with pullback $\pi^{*}$ suppressed. These identifications are diagrammed in Figure 2.1 the vertical maps on the right are projections. The functions $\mu, s$, and $f$ of equation (2.2) are defined on $\left(t_{1}, t_{2}\right)$, while $\varphi$ and other functions of geometric interest are defined on $I$.

Two instances of identification by $\pi$ deserve immediate mention. First, the Euler vector field $-J X$ on $L^{\prime}$ pushes forward to $\varphi(\tau) \frac{\partial}{\partial \tau}$ on $I \times M$, as in equation (2.18) below. The second example arises from the 1-parameter family $\omega_{M}-\tau \gamma$ of Kähler forms on $M$, regarded as a $(1,1)$-form $\omega_{M}(\tau)$ on $I \times M$, see Figure 2.2 The family of Ricci forms $\rho_{M}(\tau)$, and scalar curvature functions $\sigma_{M}(\tau)$, are similarly regarded as living on $I \times M$. These tensors depend only on the horizontal data. If a profile is specified in addition, then there is a map $\pi: L^{\prime} \rightarrow I \times M$ as above, and each of these tensors is identified with an $S^{1}$-invariant tensor on $L^{\prime}$.

The Laplacian, Ricci and scalar curvature. It is often preferable to work with endomorphisms rather than $(1,1)$-forms. 
Definition 2.1. The curvature endomorphism B is $\omega_{M}^{-1} \gamma$, the symmetric endomorphism of $T^{1,0} M$ obtained by raising the second index of $\gamma$. Similarly the Ricci endomorphism $\varrho$ of $T^{1,0} M$ is defined by raising the second index of the Ricci form $\rho_{M}$.

Put

$$
\begin{aligned}
& Q(\tau)=\frac{\omega_{M}(\tau)^{m}}{\omega_{M}^{m}}=\operatorname{det}(\mathrm{I}-\tau \mathrm{B}) \\
& R(\tau)=\operatorname{tr}_{\omega_{M}(\tau)} \rho_{M}=\operatorname{tr}\left[(\mathrm{I}-\tau \mathrm{B})^{-1} \varrho\right] \\
& P(\tau)=2 Q R(\tau)
\end{aligned}
$$

Note that $P$ and $Q$ are smooth functions on $I \times M$, with polynomial dependence upon $\tau$, while $R$ may be thought of as a rational function on $I$, with coefficients depending smoothly on $z$ in $M$. The notation reflects the fact that when the horizontal data are $\sigma$-constant, these functions depend only on $\tau$, i.e. are constant on $M$ for every $\tau \in I$.

On a Kähler manifold, if $g_{i \bar{\jmath}}$ are the components of the metric in local holomorphic coordinates, then the Ricci form is given locally by $\rho=-\sqrt{-1} \partial \bar{\partial} \log V$, where $V=\operatorname{det}\left(g_{i \bar{\jmath}}\right)$. For a bundle-adapted metric, there is a choice of coordinates such that the quantities $V_{\varphi}$ and $V_{M}$ satisfy

$$
V_{\varphi}=(\varphi Q)(\tau) V_{M}
$$

The Ricci form of $\omega_{\varphi}$ is therefore given by

$$
\rho_{\varphi}=p^{*} \rho_{M}-\sqrt{-1} \partial \bar{\partial} \log \varphi Q(\tau),
$$

and the scalar curvature is found by taking the trace:

$$
\sigma_{\varphi}=R(\tau)-\square_{\varphi} \log \varphi Q(\tau)
$$

$\square_{\varphi}$ being the $\bar{\partial}$-Laplacian of $\omega_{\varphi}$. This Laplacian has a reasonably pleasant expression in terms of $\square_{\omega_{M}(\tau)}$; for each smooth function $\psi$ on $I \times M$,

$$
\square_{\varphi} \psi=\square_{\omega_{M}(\tau)} \psi(\tau, \cdot)+\frac{1}{2 Q} \frac{\partial}{\partial \tau}\left[\varphi Q(\tau) \frac{\partial \psi}{\partial \tau}\right] .
$$

Applying this to the function $\psi=\log (\varphi Q)$ and combining with (2.10) yields

$$
\sigma_{\varphi}=R(\tau)-\square_{\omega_{M}(\tau)} \log Q(\tau)-\frac{1}{2 Q} \frac{\partial^{2}}{\partial \tau^{2}}(\varphi Q)(\tau),
$$

since $\square_{\omega_{M}(\tau)} \log \varphi Q(\tau)=\square_{\omega_{M}(\tau)} \log Q(\tau), \varphi$ being independent of $z \in M$. The first two terms together make up the scalar curvature $\sigma_{M}(\tau)$ of $\omega_{M}(\tau)$, so

$$
\sigma_{\varphi}=\sigma_{M}(\tau)-\frac{1}{2 Q} \frac{\partial^{2}}{\partial \tau^{2}}(\varphi Q)(\tau)
$$

If the data are $\sigma$-constant, (2.9) and (2.12) simplify as follows:

$$
\begin{aligned}
\rho_{\varphi} & =p^{*} \rho_{M}+\frac{1}{2 Q}(\varphi Q)^{\prime}(\tau) p^{*} \gamma-\frac{1}{2 \varphi}\left[\frac{1}{Q}(\varphi Q)^{\prime}\right]^{\prime}(\tau) d \tau \wedge d^{c} \tau \\
\sigma_{\varphi} & =R(\tau)-\frac{1}{2 Q} \frac{\partial^{2}}{\partial \tau^{2}}(\varphi Q)(\tau) .
\end{aligned}
$$


These, together with the expression

$$
\omega_{\varphi}=p^{*} \omega_{M}-\tau p^{*} \gamma+\frac{1}{\varphi} d \tau \wedge d^{c} \tau,
$$

are the main formulae that will be needed in Section 3 in the construction of metrics with prescribed scalar curvature by ODE methods.

Conventions and calculations. The wedge product is normalized so that interior multiplication is a (graded) derivation: If $V$ is a vector and $\xi$ and $\eta$ are 1-forms, then $i_{V}(\xi \wedge \eta)=\langle V, \xi\rangle \eta-\langle V, \eta\rangle \xi$. Extensive use will be made of the real operators $d=\partial+\bar{\partial}$ and $d^{c}=\sqrt{-1}(\bar{\partial}-\partial)$, and of the useful formulae

$$
d d^{c} u=2 \sqrt{-1} \partial \bar{\partial} u, \quad d u \wedge d^{c} u=2 \sqrt{-1} \partial u \wedge \bar{\partial} u,
$$

that hold for every smooth function $u$. Finally if $g$ has components $\left(g_{i \bar{\jmath}}\right)$ in local holomorphic coordinates $\left(z^{i}\right)$, then $\omega=\frac{\sqrt{-1}}{2} g_{i \bar{j}} d z^{i} \wedge d \bar{z}^{j}$.

Most of the calculations described in the previous section are best performed in a line bundle chart, namely, a local coordinate system $z^{0}, z^{1}, \ldots, z^{m}$ for $L$ in which $z^{0}=\rho e^{i \theta}$ is a fibre coordinate and $z=\left(z^{1}, \ldots, z^{m}\right)$ are (pullbacks of) coordinates on $M$. In such a chart, there is a smooth, positive function $h: V \rightarrow \mathbf{R}$ such that $r=z^{0} \bar{z}^{0} h(z)$; under change of chart, the local function $h$ is multiplied by the norm squared of a non-vanishing local holomorphic function in $V$. In a line bundle chart, the Euler vector field is given by

$$
\Upsilon=z^{0} \frac{\partial}{\partial z^{0}}=\frac{1}{2}\left(\rho \frac{\partial}{\partial \rho}-\sqrt{-1} \frac{\partial}{\partial \theta}\right),
$$

while twice the imaginary and real parts are described variously as

$$
\begin{aligned}
& X=-2 \operatorname{Im} \Upsilon=\sqrt{-1}(\Upsilon-\bar{\Upsilon})=\frac{\partial}{\partial \theta}, \\
& H=-J X=2 \operatorname{Re} \Upsilon=(\Upsilon+\bar{\Upsilon})=\rho \frac{\partial}{\partial \rho} .
\end{aligned}
$$

In particular this gives a local formula for the normalized generator $X$ of the $S^{1}$ action on $L$. It is sometimes convenient to use the fibre coordinate $w^{0}=\log z^{0}=$ : $\zeta+i \theta$, in which case $\Upsilon=\partial / \partial w^{0}$.

The level sets of $r$ are real hypersurfaces in $L$, and their tangent spaces are the horizontal spaces of the Hermitian connection $\Theta=\partial \log r=2 \partial t$ of $(L, h)$. For each point $x$ of $M$, there exists a line bundle chart $\left(z^{0}, z\right)$ such that $z^{\alpha}(x)=0$ for $1 \leq \alpha \leq m$ and $\partial_{\alpha} r=0$ on the fibre $L_{x}$. Such a coordinate system is said to be adapted to $(L, h)$ at $x \in M$. In adapted coordinates at $x$, the connection form $\Theta$ is equal to $d w^{0}=d z^{0} / z^{0}$ along the fibre $L_{x}$.

The curvature form $\gamma$ of an Hermitian line bundle satisfies

$$
-d d^{c} t=-\sqrt{-1} \partial \bar{\partial} \log r=p^{*} \gamma .
$$

Combining this with the chain rule gives the simple but important formula

$$
d d^{c} u(t)=u^{\prime \prime}(t) d t \wedge d^{c} t-u^{\prime}(t) p^{*} \gamma
$$

when $u$ is a smooth function of one variable. This is often used in the form

$$
d d^{c} \psi(\tau)=-\left(\varphi \psi^{\prime}\right)(\tau) p^{*} \gamma+\frac{1}{\varphi}\left(\varphi \psi^{\prime}\right)^{\prime}(\tau) d \tau \wedge d^{c} \tau,
$$

which follows from (2.17) and the first of (2.3). 
Referring to the momentum construction, especially formulae (2.1)-(2.4), it must be shown that $g_{\varphi}$ is a Kähler metric (namely that $\omega_{\varphi}>0$ ), and that the moment map is given by $\tau$. Expanding $d d^{c} f$ using (2.18) gives (2.16):

$$
\begin{aligned}
\omega_{\varphi} & =p^{*} \omega_{M}-\tau p^{*} \gamma+\varphi d t \wedge d^{c} t \\
& =p^{*} \omega_{M}-\tau p^{*} \gamma+\frac{1}{\varphi} d \tau \wedge d^{c} \tau
\end{aligned}
$$

Since $\omega_{M}-\tau \gamma$ and $\varphi$ are both positive on $I$ by hypothesis, it follows that $\omega_{\varphi}$ is positive. Now

$$
i_{X}(d t)=0=i_{X}\left(\omega_{M}(\tau)\right)
$$

so $i_{X} \omega_{\varphi}=-\varphi d t \wedge i_{X}\left(d^{c} t\right)=-\varphi d t$ from the formula

$$
d^{c} t=\frac{\sqrt{-1}}{2}\left(\frac{d \bar{z}^{0}}{\bar{z}^{0}}-\frac{d z^{0}}{z^{0}}\right)+\frac{1}{2} d^{c} \log h
$$

in a line bundle chart. But $\varphi d t=d \tau$ by (2.3), so $\tau$ is a choice of moment map. Changing $\tau_{0}$ adds a constant to $t$ (and changes $L^{\prime}$ by a homothety), but does not change the isometry class of $\omega_{\varphi}$.

In adapted coordinates,

$$
\omega_{\varphi}=\frac{\sqrt{-1}}{2}\left(\frac{\varphi(\tau)}{2 z^{0} \bar{z}^{0}} d z^{0} \wedge d \bar{z}^{0}+\left[g_{M}(\tau)\right]_{\alpha \bar{\beta}} d z^{\alpha} \wedge d \bar{z}^{\beta}\right)
$$

along a fibre. In terms of globally defined functions, the metric splits along a fibre into

$$
\omega_{\text {fibre }}+\omega_{\text {horiz }}=d s \wedge d^{c} s+\omega_{M}(\tau) .
$$

The expression (2.5) for the fibre metric follows at once, as does the fact that $s$ is the geodesic distance in the fibre.

The Ricci form. Equations (2.8)-(2.16) giving the volume and Ricci forms, Laplacian on functions, and scalar curvature, follow from repeated application of (2.18) and (2.3). From (2.19), the volume form is

$$
\frac{\omega_{\varphi}^{m+1}}{(m+1) !}=(\varphi Q)(\tau) \operatorname{det}\left[\left(g_{M}\right)_{\alpha \bar{\beta}}\right] \frac{1}{z^{0} \bar{z}^{0}}\left(\frac{\sqrt{-1}}{2}\right)^{m+1} \prod_{i=0}^{m} d z^{i} \wedge d \bar{z}^{i},
$$

from which (2.8) follows immediately.

Denote by $d_{M}$ and $d_{M}^{c}$ the $d$ and $d^{c}$-operators on $M$; it is necessary here to distinguish them from the corresponding operators on the total space of $L$, which we continue to denote by $d$ and $d^{c}$. For each smooth function $u$ on $I \times M$,

$$
d d^{c} u=d_{M} d_{M}^{c} u-\left[\varphi \frac{\partial u}{\partial \tau}\right] \gamma+\frac{1}{\varphi} \frac{\partial}{\partial \tau}\left[\varphi \frac{\partial u}{\partial \tau}\right] d \tau \wedge d^{c} \tau+\text { cross-terms. }
$$

(The cross-terms take the form $\bar{\partial} \tau \wedge \partial_{M}(\partial u / \partial \tau)+$ complex conjugate.) In order to take the trace with respect to $\omega_{\varphi}$, either work locally or multiply by $\omega_{\varphi}^{m}$. For the latter,

$$
\omega_{\varphi}^{m}=\omega_{M}(\tau)^{m}+m \omega_{M}(\tau)^{m-1} \cdot \frac{1}{\varphi} d \tau \wedge d^{c} \tau .
$$


Wedging with $d d^{c} u$, the cross-terms drop out, so upon division by $\omega_{\varphi}^{m+1}$,

$$
\square_{\varphi} u=\square_{\omega_{M}(\tau)} u(\tau, \cdot)+\frac{1}{2}\left(\frac{\partial}{\partial \tau}\left[\varphi \frac{\partial u}{\partial \tau}\right]-\left(\operatorname{tr}_{\omega_{M}(\tau)} \gamma\right) \varphi \frac{\partial u}{\partial \tau}\right) .
$$

The right-hand side of this is simplified by the following observation: If the eigenvalues of B at $z \in M$ are denoted $\beta_{\nu}(z)$, for $\nu=1, \ldots, m$, then $Q(\tau)=\prod_{\nu}\left[1-\tau \beta_{\nu}(z)\right]$, SO

$$
\operatorname{tr}_{\omega_{M}(\tau)} \gamma=\operatorname{tr}\left[(\mathrm{I}-\tau \mathrm{B})^{-1} \mathrm{~B}\right]=\sum_{\nu=1}^{m} \frac{\beta_{\nu}(z)}{1-\tau \beta_{\nu}(z)}=-\frac{\partial}{\partial \tau}(\log Q) .
$$

Equations (2.11) and (2.12) follow immediately, while (2.13) follows from the observation

$$
\square_{\omega_{M}(\tau)} \log \varphi Q=\square_{\omega_{M}(\tau)}(\log \varphi+\log Q)=\square_{\omega_{M}(\tau)} \log Q
$$

( $\varphi$ being pulled back by the first projection $I \times M \rightarrow I$ ) and the formulae

$$
\begin{aligned}
-\sqrt{-1} \partial_{M} \bar{\partial}_{M} \log Q & =\rho_{\omega_{M}(\tau)}-\rho_{M}, \\
-\square_{\omega_{M}(\tau)} \log Q & =\operatorname{tr}_{\omega_{M}(\tau)}\left(\rho_{\omega_{M}(\tau)}-\rho_{M}\right)=\sigma_{M}(\tau)-R(\tau) .
\end{aligned}
$$

2.2. Completeness and Extendability of Fibre Metrics. In this section, completeness criteria for the metric $g_{\varphi}$ are given in terms of the boundary behaviour of $\varphi$. Because each fibre is totally geodesic by Proposition 2.2 below, $g_{\varphi}$ is complete if and only if

- The metric $g_{M}(\tau)$ is complete for every $\tau \in I$, and

- The fibre metric $g_{\text {fibre }}$ is complete.

The first condition is assumed in our existence theorems, and the second condition is characterized in elementary terms by Proposition 2.3 below.

Total geodesy of fibres. Let $g$ be a bundle-adapted metric on an invariant subbundle $L^{\prime}$. It is well-known that the fibres of $L^{\prime}$ are totally geodesic with respect to $g$. Nonetheless, we reproduce a proof here as an excuse to calculate the LeviCivita connection of $g$.

Proposition 2.2. Let $g_{\varphi}$ be a bundle-adapted metric on $L^{\prime} \subset L$. Then each fibre of $L^{\prime}$ is totally geodesic with respect to $g_{\varphi}$.

Proof. It suffices to calculate the Levi-Civita connection $D$ of $g_{\varphi}$ and show that $D_{\partial_{0}} \partial_{0}$ is tangent to the fibre. Let $\left(z^{0}, z\right)$ be a line bundle chart, $w^{0}=\log z^{0}$, and let $\partial_{\alpha}$ denote partial differentiation. Recall that the connection form of $(L, h)$ is equal to $\Theta=\partial \log r$. With respect to the coordinates $\left(w^{0}, z\right)$, the vector-valued $(1,0)$-form $\Theta$ is given by the column

$$
\left[\Theta_{i}\right]^{\mathrm{t}}=\left[\begin{array}{ll}
1 & \Theta_{\alpha}
\end{array}\right]^{\mathrm{t}}, \quad \Theta_{\alpha}=h^{-1} \partial_{\alpha} h,
$$

and $\bar{\partial}\left(\Theta_{\alpha} d z^{\alpha}\right)=p^{*} \gamma$. The components of $g_{\varphi}$ are given by the Hermitian $(1+m) \times$ $(1+m)$ block matrix

$$
G=\left[\begin{array}{ll}
g_{0 \overline{0}} & g_{0 \bar{\beta}} \\
g_{\alpha \overline{0}} & g_{\alpha \bar{\beta}}
\end{array}\right]=2 \varphi(\tau)\left[\begin{array}{cc}
1 & {\left[\Theta_{\bar{\beta}}\right]^{\mathrm{t}}} \\
{\left[\Theta_{\alpha}\right]} & {\left[\Theta_{\alpha}\right]\left[\Theta_{\bar{\beta}}\right]^{\mathrm{t}}}
\end{array}\right]+\left[\begin{array}{cc}
0 & 0 \\
0 & {\left[g_{M}(\tau)\right]_{\alpha \bar{\beta}}}
\end{array}\right] .
$$


The inverse matrix $G^{-1}$ is found by (block) row-reduction to be

$$
\frac{1}{2 \varphi(\tau)}\left[\begin{array}{ll}
1 & 0 \\
0 & 0
\end{array}\right]+\left[\begin{array}{rr}
{\left[\Theta_{\bar{\beta}}\right]^{\mathrm{t}}\left[g_{M}(\tau)\right]^{\bar{\beta} \alpha}\left[\Theta_{\alpha}\right]} & -\left[\Theta_{\bar{\beta}}\right]^{\mathrm{t}}\left[g_{M}(\tau)\right]^{\bar{\beta} \alpha} \\
-\left[g_{M}(\tau)\right]^{\bar{\beta} \alpha}\left[\Theta_{\alpha}\right] & {\left[g_{M}(\tau)\right]^{\bar{\beta} \alpha}}
\end{array}\right] .
$$

The Levi-Civita connection form of $g$ is represented in a line bundle chart by the matrix-valued $(1,0)$-form $G^{-1} \partial G$. A short calculation shows that in adapted coordinates,

$$
G^{-1} \partial G=\left[\begin{array}{cc}
(1 / 2) \varphi^{\prime}(\tau) \partial t & \partial\left[\Theta_{\bar{\beta}}\right]^{\mathrm{t}} \\
\varphi(\tau)\left[g_{M}(\tau)\right]^{\bar{\beta} \alpha} \partial\left[\Theta_{\alpha}\right] & {\left[g_{M}(\tau)\right]^{\bar{\beta} \nu}\left[g_{M}(\tau)\right]_{\alpha \bar{\beta}}}
\end{array}\right]
$$

along the fibre. Evaluating on the tangent vector $\partial_{0}=\partial / \partial w^{0}$ at a point of the fibre gives the representation (with respect to the frame $\left\{\partial_{i}\right\}_{i=0}^{n}$ ) of the covariant derivative $D_{\partial_{0}}$ in the fibre direction. The first column is $\left[\begin{array}{lllll}(1 / 2) \varphi^{\prime}(\tau) & 0 & \cdots & 0\end{array}\right]^{t}$, implying the covariant derivative $D_{\partial_{0}} \partial_{0}$ is tangent to the fibre, so the fibre is totally geodesic.

Completeness of the fibre metric. By equation (2.2), which determines the geodesic distance function $s(t): L^{\times} \rightarrow \mathbf{R}$ in terms of the profile, a simple completeness criterion is easily deduced by expanding the profile as a series near an endpoint of $I$. Completeness is guaranteed by divergence of the $s$ integral, though divergence at a finite endpoint (corresponding to an end of finite area in the fibre) is not necessary since the fibre metric may extend smoothly to the origin (or to the point at infinity). For simplicity, Proposition 2.3 is stated for profiles asymptotic to an integer power of $\tau$ at the endpoint(s) of $I$ (or as $\tau \rightarrow \infty$ ). This is no loss for present purposes, since the profiles of greatest interest are rational functions, which arise when $g$ has constant scalar curvature (or is formally extremal).

Proposition 2.3. Let $\varphi: I \rightarrow \mathbf{R}$ be a profile that has a zero or pole (of integer order) at each endpoint of $I$. Then the associated fibre metric is complete if and only if one of the following conditions holds at each endpoint of I:

- Finite Endpoint $(s)$

(i): The profile $\varphi$ vanishes to first order, and $\left|\varphi^{\prime}\right|=2$ at the endpoint; or

(ii): The profile vanishes to order at least two.

- Infinite Endpoint(s)

(iii): The profile grows at most quadratically: There is a $K$ such that $\varphi(\tau) \leq$ $K \tau^{2}$ for $|\tau| \gg 0$.

Proof. Most of these conclusions are immediate from (2.2) upon expansion of the profile as a series. The one point requiring a small amount of work is to determine what happens when the profile vanishes to order one at a finite endpoint of $I$.

In a line bundle chart, $r=h\left|z^{0}\right|^{2}$, and by (2.5) the fibre metric is

$$
g_{\text {fibre }}=\varphi(\tau)\left|\frac{d z^{0}}{z^{0}}\right|^{2}=\left[\frac{\varphi(\tau)}{r}\right] h\left|d z^{0}\right|^{2} .
$$

Since the horizontal part is smooth, $g_{\varphi}$ is smooth at $r=0$ if and only if $\varphi(\tau) / r$ has a finite, positive limit as $\tau \rightarrow 0$. (This limit makes sense, for (2.2) gives $t$ and hence $r$ as a function of $\tau$.) Using (2.2) to find $t$, with $\varphi(\tau)=a_{1} \tau+O\left(\tau^{2}\right)$, gives $\log \tau=$ constant $+a_{1} t+\cdots$, or $\tau=a r^{a_{1} / 2}+\cdots$ for some $a>0$. Thus $\varphi(\tau) / r$ has 
a finite, positive limit as $r \rightarrow 0$ if and only if $a_{1}=2$, or equivalently $\varphi(0)=0$ and $\varphi^{\prime}(0)=2$. A similar argument takes care of a finite upper endpoint.

Geometry of fibre metrics. This section is intended provide a pleasant digression by linking the geometry of fibre metrics (given by momentum profiles) with familiar $S^{1}$-invariant metrics, both abstractly and as surfaces of revolution in $\mathbf{R}^{3}$. The results are summarized in Table 2.1, which shows clearly how much simpler these metrics look in 'momentum coordinates' than in the standard complex coordinate $z$. Verification of the entries in Table 2.1 requires only repeated application of (2.2). We include this table mainly for pedagogical reasons: to indicate concretely how the geometry of the fibre metric is related to the profile, and to emphasize that the image $I$ of the moment map $\tau$ is essentially independent from the image of the function $r=|z|^{2}=e^{2 t}$.

In (vi) the annulus is determined, up to conformal equivalence, by the ratio of the inner and outer radii and hence by $c \alpha$. In examples (i)-(iii), with $\varphi^{\prime}(0)=2$, metrics with conical singularity at the origin arise by changing the coefficient of $\tau$.

To compare these metrics with surfaces of revolution in $\mathbf{R}^{3}$, let $\xi$ be a positive function. Upon revolution about the $y$-axis, the area and length elements satisfy

$$
d \tau=\xi(y) \sqrt{1+\xi^{\prime}(y)^{2}} d y, \quad d s=\sqrt{1+\xi^{\prime}(y)^{2}} d y .
$$

The profile gives the length squared of $\frac{\partial}{\partial \theta}$, namely $\varphi(\tau)=\xi(y)^{2}$. Thus

$$
\varphi^{\prime}(\tau)=\frac{2 \xi^{\prime}(y)}{\sqrt{1+\xi^{\prime}(y)^{2}}}, \quad \text { or } \quad \xi^{\prime}(y)=\frac{\varphi^{\prime}(\tau)}{\sqrt{4-\varphi^{\prime}(\tau)^{2}}},
$$

which implies $\left|\varphi^{\prime}(\tau)\right| \leq 2$, with equality if and only if $\left|\xi^{\prime}(y)\right|=\infty$ : The fibre metric embeds as a surface of revolution if and only if the profile is not too steep. This confirms the well-known facts that a portion of the cusp (iv) - corresponding to the momentum sub-interval $\left(0,1 / c^{2}\right)$ - embeds as a surface of revolution in $\mathbf{R}^{3}$ (the pseudosphere), while no invariant domain of the Poincaré disc (iii) embeds in this way.

It is also worth noting that intuition deriving from surfaces of revolution can be misleading. Taking $I=[0, \infty)$ and $\varphi(\tau)=2 \tau+\tau^{3}$ gives an incomplete metric of infinite area on the disk!

\section{Constant Scalar Curvature Metrics of Infinite Volume}

This section is devoted to the proofs of Theorems B, C, and D Section 3.1 deals with Theorems $\mathrm{B}$ and $\mathrm{C}$ while Section 3.2 with Theorem $\mathrm{D}$. The proofs are separated in this way because of the behaviour of the family of Kähler forms $\omega_{M}(\tau)=$ $\omega_{M}-\tau \gamma$; in Theorems B and C, $\omega_{M}(0)$ is non-degenerate, whereas in Theorem D the family $\left\{\omega_{M}(\tau)\right\}$ drops rank at $\tau=0$, leading to a metric on a partial blow-down of $L$.

3.1. Metrics on Line Bundles. Let $\left\{p:(L, h) \rightarrow\left(M, \omega_{M}\right), I\right\}$ be $\sigma$-constant horizontal data with $\gamma \prec 0$, and with $I=[0, \infty)$ or $(0, \infty)$. In particular, $\omega_{M}(\tau)$ is a Kähler form on $M$ for all $\tau \geq 0$, and the metric $g_{M}(\tau)$ is complete since $g_{M}$ is assumed to be complete and $-\tau \gamma$ is positive semidefinite for $\tau \in I$. As in Section 2 


\begin{tabular}{|c|c|c|c|c|c|c|}
\hline 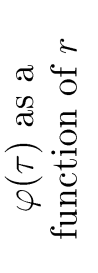 & 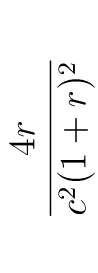 & $\therefore$ & 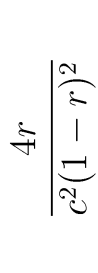 & 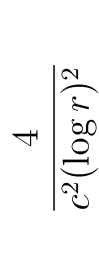 & $\mathcal{Z}_{\partial}$ & 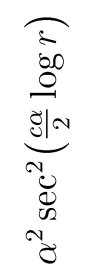 \\
\hline 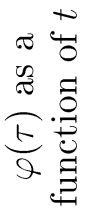 & 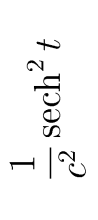 & స్ & 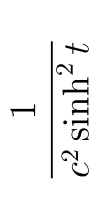 & -||$_{\mathcal{N}}^{\mathcal{N}}$ & $\mho_{\partial}$ & 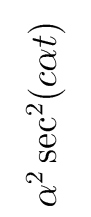 \\
\hline 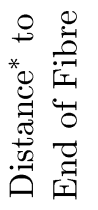 & 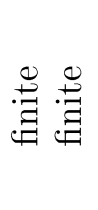 & 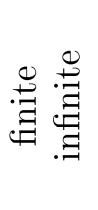 & 总. 莺 & 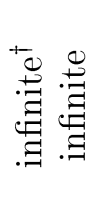 & 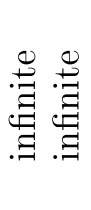 & 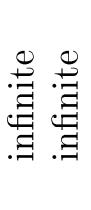 \\
\hline \begin{tabular}{l}
$\infty$ \\
$\infty$ \\
\multirow{\pi}{*}{} \\
\multirow{\sim}{*}{} \\
+
\end{tabular} & $\begin{array}{l}8 \\
8 \\
1 \\
\end{array}$ & $\begin{array}{l}8 \\
8 \\
1 \\
\end{array}$ & $\begin{array}{l}0 \\
8 \\
1\end{array}$ & $\begin{array}{l}0 \\
8 \\
1\end{array}$ & $\begin{array}{l}8 \\
8 \\
1\end{array}$ & 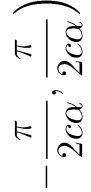 \\
\hline 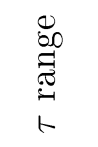 & $\begin{array}{l}\bar{T} \\
\stackrel{\nu}{u} \\
\sim \\
\tilde{0}\end{array}$ & $\begin{array}{l}8 \\
0 \\
0\end{array}$ & $\begin{array}{l}8 \\
0\end{array}$ & $\begin{array}{l}8 \\
8 \\
0\end{array}$ & $\tilde{\mu}_{1}$ & $\tilde{\sim}_{1}$ \\
\hline$\frac{\overparen{E}}{a}$ & 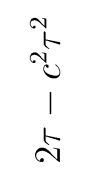 & $\stackrel{5}{\circ}$ & 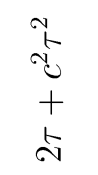 & $\begin{array}{l}\text { to } \\
\text { if }\end{array}$ & $\widetilde{\gamma}$ & 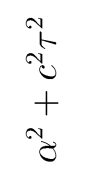 \\
\hline 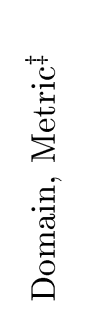 & 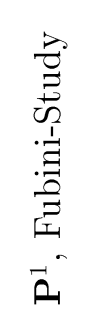 & 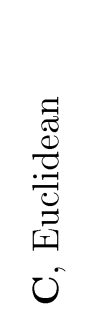 & 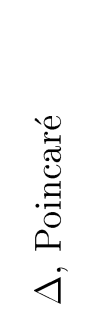 & 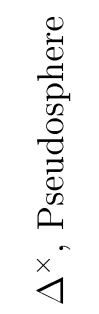 & 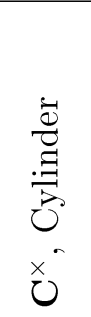 & $\begin{array}{l}\stackrel{0}{\Xi} \\
\vdots \\
\Xi \\
\Xi\end{array}$ \\
\hline & 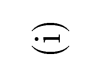 & $\overparen{\Xi}$ & $\overparen{\overbrace \Xi}$ & 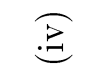 & $B$ & $\overparen{E}$ \\
\hline
\end{tabular}

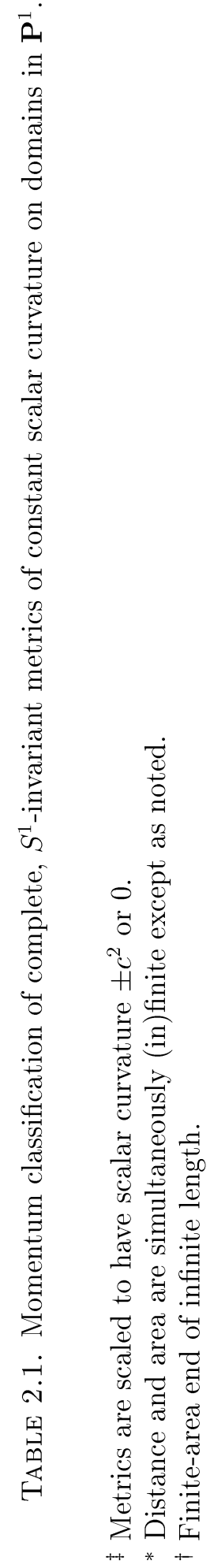


define functions $Q, R: I \rightarrow \mathbf{R}$ by

$$
Q(\tau)=\operatorname{det}(1-\tau \mathrm{B}), \quad R(\tau)=\operatorname{tr}\left((1-\tau \mathrm{B})^{-1} \varrho\right) .
$$

Because the data are $\sigma$-constant, $Q(\tau)$ is the (constant) scale factor for the volume form of $\omega_{M}(\tau)$ relative to $\omega_{M}$ and is a polynomial in $\tau$ with only negative roots, while $R(\tau)=\sigma_{M}(\tau)$ is the scalar curvature of $g_{M}(\tau)$ and is a rational function that is bounded below on $I$. Indeed, $R$ has an asymptotic value $R(\infty)$ as $\tau \rightarrow \infty$, which may be interpreted as the trace of $\varrho$ restricted to the 0 -eigenbundle of B. By Corollary A.1, the scalar curvature of $\omega_{\varphi}$ is

$$
\sigma_{\varphi}=R(\tau)-(1 / 2 Q)(\varphi Q)^{\prime \prime}(\tau)
$$

Now let $\sigma$ be a function on $I$. The problem of prescribing the scalar curvature of $\omega_{\varphi}$ is given by the equation $\sigma=\sigma_{\varphi}$, which has the solution

$$
(\varphi Q)(\tau)=(\varphi Q)(0)+(\varphi Q)^{\prime}(0) \tau+2 \int_{0}^{\tau}(\tau-x)(R(x)-\sigma(x)) Q(x) d x
$$

in terms of the initial data $\varphi(0)$ and $\varphi^{\prime}(0)$. The momentum construction yields a metric of infinite fibre area if and only if $\varphi$ is positive on $(0, \infty)$. This metric is complete if $\varphi$ grows at most quadratically at $\infty$ and satisfies the boundary conditions given in Proposition 2.3 $\varphi(0)=0$, and either $\varphi^{\prime}(0)=2$ or $\varphi^{\prime}(0)=0$. The first case is the one needed for Theorem $\mathrm{B}$, the second for Theorem $\mathrm{C}$. These will now be considered in turn.

Proof of Theorem B. Setting $\sigma=c$ (constant) and using the initial conditions $\varphi(0)=0, \varphi^{\prime}(0)=2$ in 3.2 gives

$$
\varphi(\tau)=\frac{2}{Q(\tau)}\left(\tau+\int_{0}^{\tau}(\tau-x)(R(x)-c) Q(x) d x\right) .
$$

The notation $\varphi_{c}(\tau)$ or $\varphi(\tau, c)$ will be used when the dependence of the profile on $c$ is being emphasized. Define the set $\mathcal{C} \subset \mathbf{R}$ of "allowable scalar curvatures" by

$$
\mathcal{C}=\left\{c \in \mathbf{R} \mid \varphi_{c}(\tau)>0 \text { for all } \tau>0\right\} .
$$

In words, $\mathcal{C}$ is the set of $c$ for which equation (3.3) defines a momentum profile on $I$.

Lemma 3.1. There is a $c_{0} \in \mathbf{R}$ such that either $\mathcal{C}=\left(-\infty, c_{0}\right)$ or $\mathcal{C}=\left(-\infty, c_{0}\right]$.

Proof. The function $R$ is bounded below on $I$, so the integrand in (3.3) is positive for $c \ll 0$, implying $\varphi_{c}>0$ on $(0, \infty)$ for $c \ll 0$. In particular, $\mathcal{C}$ is non-empty. If $\tau>0$, then

$$
\frac{\partial \varphi}{\partial c}(\tau, c)=-\frac{2}{Q(\tau)} \int_{0}^{\tau}(\tau-x) Q(x) d x<0,
$$

so $\varphi(\tau, c)$ is strictly decreasing with respect to $c$. Consequently, if $c \in \mathcal{C}$ and $c^{\prime}<c$, then $c^{\prime} \in \mathcal{C}$. Finally, $\mathcal{C}$ is bounded above since by (3.3), $\varphi_{c}$ is not everywhere positive for $c>R(\infty)$. In summary, $\mathcal{C}$ is a half-line, unbounded below. Set $c_{0}=$ $\sup \mathcal{C} \leq R(\infty)$.

Since the initial condition ensures that $\varphi_{c}$ is positive for sufficiently small positive $\tau$, the momentum construction yields a metric which for notational convenience will be denoted $g_{c}$.

Lemma 3.2. If $c<c_{0}$ then $g_{c}$ is a complete metric on $\Delta(L)$, and $g_{c_{0}}$ is a complete metric on $L$. 
Proof. By Proposition [2.3, completeness of the metric is equivalent to 'at most quadratic' growth of $\varphi_{c}$ at $\infty$, provided the profile is positive on $(0, \infty)$. To establish the growth condition, it is easiest to write $R-c$ as a constant plus a rational function $R_{0}$ vanishing at $\infty$ :

$$
R(\tau)-R(\infty)=: R_{0}(\tau), \quad c-R(\infty)=: \tilde{c},
$$

so $R(\tau)-c=R_{0}(\tau)-\tilde{c}$. Define polynomials

$$
P_{1}(\tau)=\tau+\int_{0}^{\tau}(\tau-x) R_{0}(x) Q(x) d x, \quad P_{2}(\tau)=\int_{0}^{\tau}(\tau-x) Q(x) d x
$$

Because $R_{0}$ vanishes at $\infty, \operatorname{deg} P_{1} \leq 1+\operatorname{deg} Q$, and $\operatorname{deg} P_{2}=2+\operatorname{deg} Q$. From (3.3),

$$
\varphi(\tau, c)=\frac{2}{Q(\tau)}\left(P_{1}(\tau)-\tilde{c} P_{2}(\tau)\right),
$$

so if $\tilde{c}=0$, then $\varphi_{c}(\tau) \leq K \tau$ as $\tau \rightarrow \infty$, while $\varphi_{c}(\tau) \sim K \tau^{2}$ if $\tilde{c}<0$, namely if $c<R(\infty)$. Since $c_{0} \leq R(\infty)$, it follows that each profile $\varphi_{c}$ with $c<c_{0}$ gives rise to a complete fibre metric, hence to a complete metric $g_{c}$. Because $\varphi_{c}$ grows quadratically, the $t$ integral converges as $\tau \rightarrow \infty$, so up to homothety $g_{c}$ lives on the unit disk bundle $\Delta(L)$.

It remains to investigate the borderline case. First observe that every profile $\varphi_{c}$ with $c<c_{0}$ is bounded away from zero except near $\tau=0$ (since $\varphi_{c}^{\prime}(0)=2$, and $\varphi_{c}$ is positive on $(0, \infty)$ and has infinite limit as $\tau \rightarrow \infty)$. By the proof of Lemma 3.1 $\varphi(\tau, c)$ is decreasing in $c$. Since $c_{0}$ is the supremum of $c$ for which $\varphi_{c}$ is positive on $(0, \infty)$, it follows that $\varphi_{c_{0}}$ is non-negative on $I$ by continuity of $\varphi(\tau, c)$ in $c$. The borderline profile is not identically zero, since $\varphi_{c_{0}}^{\prime}(0)=2$.

Two possibilities occur: $\varphi_{c_{0}}$ has a positive zero, or is positive on $(0, \infty)$. In the first case let $b$ be the first positive zero of $\varphi_{c_{0}}$. Then $\varphi_{c_{0}}^{\prime}(b)=0$ as well, for $\varphi_{c_{0}}$ is real-analytic and non-negative. Since $\varphi_{c_{0}}$ vanishes to order at least two, the $t$ and $s$ integrals diverge, so the associated metric lives on the total space of $L$ and is complete, but has finite-area fibres.

Consider now the second possibility, $\varphi_{c_{0}}>0$ on $(0, \infty)$. We claim in this case that $c_{0}=R(\infty)$ (i.e. $\tilde{c}=0$ ). For if not, the borderline profile is positive and grows quadratically, hence is bounded away from zero except near $\tau=0$; a glance at (3.4) shows $\tilde{c}$ may be increased slightly, preserving positivity of the profile, but this contradicts the definition of $c_{0}$. Hence $\varphi_{c_{0}}(\tau)=\left(P_{1} / Q\right)(\tau) \leq K \tau$ and again $g_{c_{0}}$ lives on $L$.

The dichotomy at $c=c_{0}$ is summarized as follows:

1. $\varphi_{c_{0}}$ has a positive zero in $I$ and yields a metric with fibrewise finite area, see Figure 3.2.

2. $\varphi_{c_{0}}$ is positive on $(0, \infty)$ and yields a complete metric of infinite fibre area, see Figure 3.1

As shown above, the second alternative implies $c_{0}=R(\infty)$ (so $c_{0}<R(\infty)$ implies the first alternative, see Figure 3.2), but this is the only general conclusion that can be drawn. Further, it is not necessarily true that $c_{0}=0$ (since generally $R(\infty) \neq 0$, for example); this issue is addressed in detail below. However, if $\gamma<0$, or if the construction yields a metric that is Einstein, then the borderline metric is scalar-flat, while the others have negative curvature. 


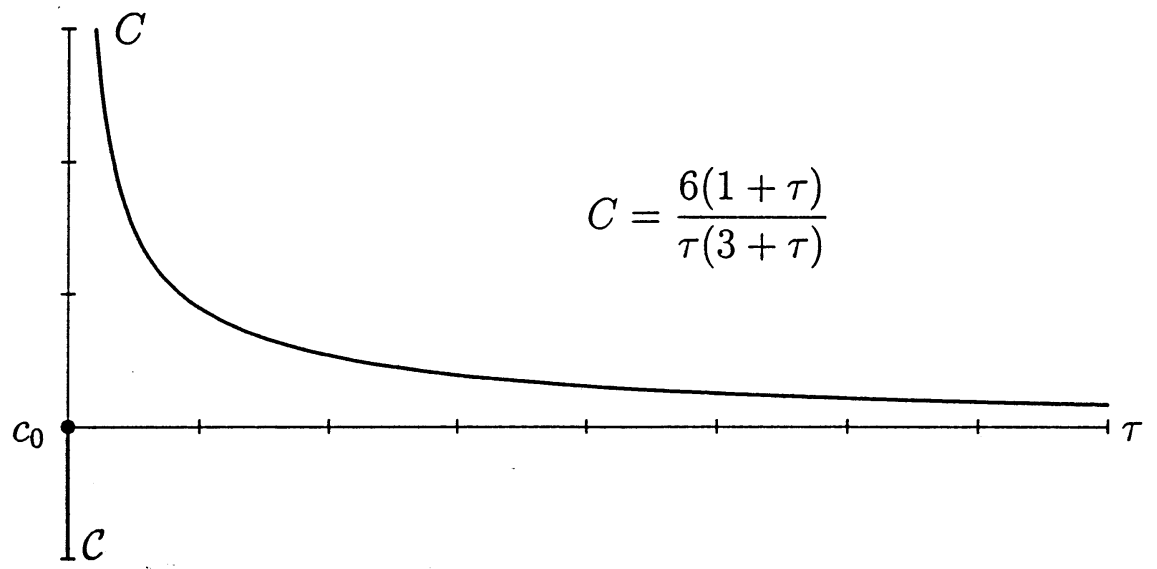

FiguRE 3.1. The rational function corresponding to the tautological bundle $\mathcal{O}_{\mathbf{P}^{1}}(-1)$. Here $\mathcal{C}=(-\infty, 0]$.

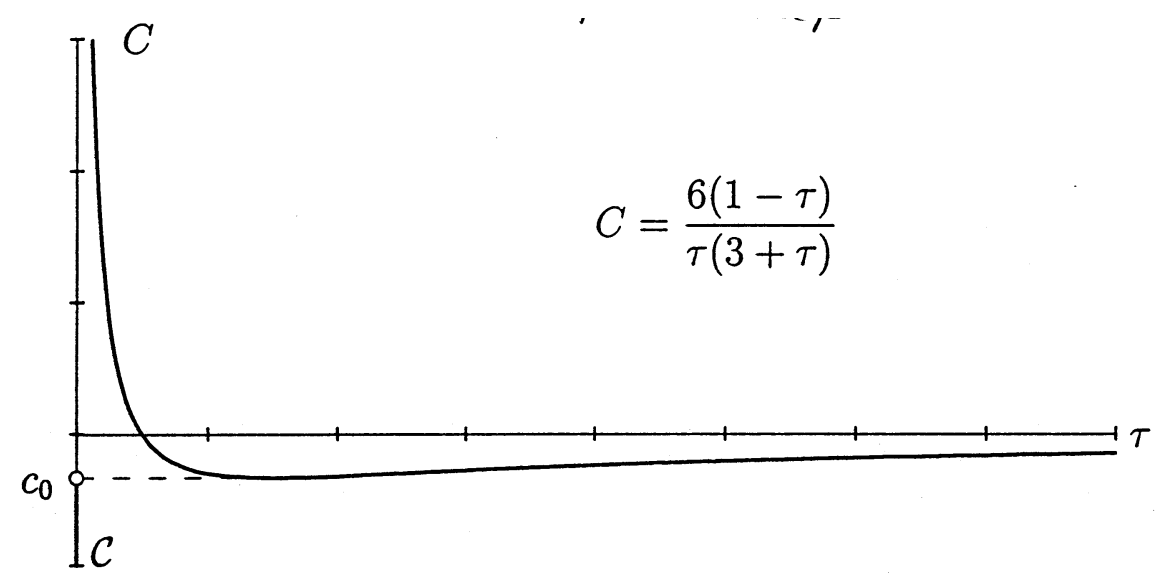

FiguRE 3.2. The rational function corresponding to a bundle of degree -1 over a curve of genus 2 .

The borderline constant $c_{0}$ has an alternative interpretation. Consider the equation $\varphi(\tau, c)=0$. From (3.4), this level set is the graph of the rational function $C: I \rightarrow \mathbf{R}$ defined by

$$
C(\tau)=R(\infty)+\frac{P_{1}(\tau)}{P_{2}(\tau)} .
$$

The degree of $P_{1}$ is less than the degree of $P_{2}$, so $C(\tau) \rightarrow R(\infty)$ as $\tau \rightarrow \infty$. Furthermore, $P_{1}$ vanishes to order one and $P_{2}$ vanishes to order two at $\tau=0$, so $C(\tau) \rightarrow+\infty$ as $\tau \rightarrow 0^{+}$. Hence the function $C$ is bounded below on $(0, \infty)$, and it follows immediately from the definition that $c_{0}=\inf \{C(\tau) \mid \tau \in(0, \infty)\}$, see Figures 3.1 (where $c_{0}=0$ is an allowable scalar curvature) and 3.2 (where $c_{0}<0$ is not an allowable scalar curvature).

If $c>c_{0}$, then $\varphi_{c}$ is not non-negative on $(0, \infty)$, so there is a first positive root $b$, and the fibre metric has finite area. Three possibilities occur: 
1. $\varphi_{c}^{\prime}(b)=0$, and the fibre metric is complete, with a cusp end at $\tau=b$;

2. $\varphi_{c}^{\prime}(b)=-2$, and the metric extends smoothly to the $\mathbf{P}^{1}$-bundle $\widehat{L}$ (but note Remark 3.5 below);

3. $\varphi_{c}^{\prime}(b) \neq 0,-2$, so the metric is incomplete and has no smooth extension.

Lemma 3.3. There are at most finitely many $c>c_{0}$ for which the profile $\varphi_{c}$ satisfies one of the first two conditions.

Proof. By (3.4), $\varphi(\tau, c)=0$ if and only if $\tilde{c}=P_{1}(\tau) / P_{2}(\tau)$. Substitution shows that if $\varphi(\tau, c)=0$, then

$$
\frac{\partial \varphi}{\partial \tau}(\tau, c)=2 \frac{P_{1}(\tau)}{Q(\tau)}\left(\log \frac{P_{1}}{P_{2}}\right)^{\prime}(\tau)
$$

This is a non-constant rational function, which takes the values 0 and -2 for at most finitely many values of $\tau$. Consequently, there are at most finitely many pairs $(\tau, c)$ satisfying

$$
\varphi(\tau, c)=0 \quad \text { and } \quad \frac{\partial \varphi}{\partial \tau}(\tau, c)=0 \text { or }-2 .
$$

In any event, a value $c>c_{0}$ does not give rise to a metric with infinite volume.

The final task is to determine when a metric just constructed is Einstein. Equation (2.16) expresses the Kähler form of $g_{\varphi}$ in terms of $\varphi$ and the horizontal data, while 2.14) similarly expresses the Ricci form. Setting $\rho_{\varphi}=\lambda \omega_{\varphi}$ gives

$$
-\left(\frac{1}{2 Q}(\varphi Q)^{\prime}\right)^{\prime}=\lambda \quad \text { and } \quad \rho_{M}+\frac{1}{2 Q}(\varphi Q)^{\prime}(\tau) \gamma=\lambda\left(\omega_{M}-\tau \gamma\right) .
$$

Integrating the first and using the initial conditions $\varphi(0)=0, \varphi^{\prime}(0)=2$,

$$
\frac{1}{2 Q}(\varphi Q)^{\prime}(\tau)=1-\lambda \tau
$$

Substituting this back into the second equation,

$$
\rho_{M}+\gamma=\lambda \omega_{M}
$$

Assume from now on that $\lambda \leq 0$, see Remark 3.5 below. Integrating (3.5), again using $\varphi(0)=0$, gives

$$
\varphi(\tau)=\frac{2}{Q(\tau)} \int_{0}^{\tau}(1-\lambda x) Q(x) d x
$$

which is clearly positive for all $\tau>0$. Completeness follows since $\varphi$ grows linearly (if $\lambda=0$ ) or quadratically (if $\lambda<0$ ), and the scalar curvature is $\lambda(m+1)$. Conversely, a bundle-adapted metric arising in this way is Einstein:

Lemma 3.4. If the horizontal data are $\sigma$-constant and satisfy $\rho_{M}+\gamma=\lambda \omega_{M}$ for some $\lambda \leq 0$, then the bundle-adapted metric with $c=\lambda(m+1)$ is Einstein-Kähler.

Proof. Write $\rho_{M}=\lambda \omega_{M}-\gamma=\lambda\left(\omega_{M}-\tau \gamma\right)-(1-\lambda \tau) \gamma$. Taking the trace with respect to $\omega_{M}(\tau)$, using the definition of $R$ and recalling that $\operatorname{tr}_{\omega_{M}(\tau)} \gamma=-\frac{\partial}{\partial \tau}(\log Q)$ by equation (2.22) immediately implies

$$
R(\tau)=\lambda m+(1-\lambda \tau) \frac{Q^{\prime}(\tau)}{Q(\tau)} .
$$


If $c=\lambda(m+1)$, then

$$
(R(\tau)-c) Q(\tau)=Q^{\prime}(\tau)-\lambda\left(Q(\tau)+\tau Q^{\prime}(\tau)\right)=\frac{d}{d \tau}((1-\lambda \tau) Q(\tau)) .
$$

Integrating twice, using $\varphi(0)=0$ and $\varphi^{\prime}(0)=2$, proves the profile (3.6) coincides with the profile (3.3).

This completes the proof of Theorem B

Remark 3.5. When the Einstein constant $\lambda$ is positive, every completion of $L^{\times}$is compact by Myer's Theorem, so there is a non-vacuous boundary condition imposed on (3.5), namely that $\varphi^{\prime}(b)=-2$ when $\varphi(b)=0$. Koiso and Sakane have shown this is equivalent to vanishing of the Futaki invariant of the compactification. This boundary condition is never satisfied if the curvature form $\gamma$ is negative semi-definite unless $\gamma=0$. To see this, observe that $\varphi(b)=0$ if and only if

$$
\int_{0}^{b}(1-\lambda x) Q(x) d x=0,
$$

in which case a short calculation shows that $\varphi^{\prime}(b)=-2$ if and only if $b \lambda=2$. Substituting back into (3.6),

$$
\begin{aligned}
\varphi(\tau) & =\frac{2}{Q(\tau)} \int_{0}^{\tau}\left(1-\frac{2}{b} x\right) Q(x) d x \\
& =-\frac{4}{b Q(\tau)} \int_{-b / 2}^{\tau-(b / 2)} x Q(x+(b / 2)) d x
\end{aligned}
$$

and this is not zero when $\tau=b$ because $Q$ is positive and increasing on the interval $[0, \infty)$ unless $\gamma=0$. It is possible for the boundary conditions to be satisfied if $\gamma>0$; for example, $\mathbf{P}^{m+1}$ is a smooth compactification of the total space of $\mathcal{O}_{\mathbf{P}^{m}}(1)$.

Proof of Theorem [C. The proof of Theorem $[$ differs from the proof of Theorem $\mathbb{B}$ in the initial conditions, and consequently in the choice of momentum interval; $\varphi^{\prime}(0)=0$ rather than $\varphi^{\prime}(0)=2$, so $I=(0, \infty)$ rather than $[0, \infty)$. The details are almost exactly parallel: The profile of equation (3.3) is replaced by

$$
\varphi(\tau)=\frac{2}{Q(\tau)} \int_{0}^{\tau}(\tau-x)(R(x)-c) Q(x) d x,
$$

which is positive for $\tau>0$ if $c \ll 0$. An interval $\mathcal{C}^{\times}$, with supremum equal to $c_{0}^{\times}$, is defined as before. The proof that the metrics are complete and live on $\Delta^{\times}(L)$ if $c<c_{0}^{\times}$is entirely analogous to the proof of Lemma 3.2. If $R(\tau)$ is non-constant, then the borderline profile is not identically zero, and induces a metric on $L^{\times}$.

Exactly as before, the metric is Einstein if and only if

$$
\rho_{M}=\lambda \omega_{M} \quad \text { and } \quad \frac{1}{2 Q}(\varphi Q)^{\prime}(\tau)=-\lambda \tau,
$$

so

$$
\varphi(\tau)=-\frac{2 \lambda}{Q(\tau)} \int_{0}^{\tau} x Q(x) d x
$$


This function $\varphi$ is positive on $(0, \infty)$ if and only if $\lambda<0$. Conversely, if $\rho_{M}=\lambda \omega_{M}$ and $c=\lambda(m+1)$ for some $\lambda<0$, then an argument analogous to the proof of Lemma 3.4 shows that

$$
(R(\tau)-c) Q(\tau)=2 \lambda \frac{d}{d \tau}(-\tau Q(\tau)),
$$

so the associated metric is Einstein.

One significant difference between Theorems $\mathrm{B}$ and $\mathrm{C}$ appears when $R$ is constant. In the event $g_{M}$ is Ricci-flat (so $R \equiv 0$ ) there is no profile satisfying the boundary conditions $\varphi(0)=\varphi^{\prime}(0)=0$ that induces a scalar-flat metric. In fact, the equation for scalar-flatness is $(\varphi Q)^{\prime \prime}=0$, while the initial conditions imply $(\varphi Q)(0)=(\varphi Q)^{\prime}(0)=0$, so $\varphi \equiv 0$. Even by relaxing the initial conditions, the only scalar-flat metrics that arise are uninteresting. To wit, the equation $(\varphi Q)^{\prime \prime}=0$ leads to the candidate profile $\varphi(\tau)=\left(a_{0}+a_{1} \tau\right) / Q(\tau)$. This function induces a complete metric only if it is everywhere positive (and perhaps has a removable discontinuity), but in this event the momentum interval is all of $\mathbf{R}$. The requirement that $\omega_{M}-\tau \gamma$ be a Kähler form for all $\tau \in \mathbf{R}$ forces $\gamma=0$, so $Q \equiv 1$ and the profile reduces to a positive constant. The induced metric is a local product of $g_{M}$ and a flat cylinder of radius equal to the value of the profile.

Bounds on $c_{0}$. To facilitate the proof of Lemma 3.3 a rational function $C$ was introduced whose infimum over $I$ is equal to $c_{0}$. Since $C$ has an explicit expression in terms of the curvature of the horizontal data, $c_{0}$ can in principle be estimated in terms of the curvature. A crude estimate comes from the following simple observations. If $R(\tau)-c \geq 0$ for all $\tau \geq 0$, then the profile (3.3) is positive for $\tau>0$, while if $R(\infty)-c<0$, then the profile is not always positive. Thus

$$
\inf _{\tau \geq 0} R(\tau) \leq c_{0} \leq R(\infty)
$$

To estimate the lower bound, pick a point $z$ of $M$ and choose an orthonormal basis of $T_{z}^{1,0} M$ relative to which B is diagonal. Denote by $\beta_{\nu}$ the eigenvalues of B, indexed so that $\beta_{1} \leq \cdots \leq \beta_{j}<0=\beta_{j+1}=\cdots=\beta_{m}$, and let $\varrho_{\nu}$ be the diagonal elements of $\varrho$ in the basis. Then

$$
R(\tau)=\frac{\varrho_{1}}{1-\beta_{1} \tau}+\cdots+\frac{\varrho_{j}}{1-\beta_{j} \tau}+\varrho_{j+1}+\cdots+\varrho_{m}
$$

Since the coefficients of $R(\tau)$ are by hypothesis independent of $z$, certain combinations of the $\varrho_{\nu}$ are independent of $z$. Indeed suppose that among the $\beta_{\nu}$ the distinct real numbers occurring are $b_{1}<\cdots<b_{\ell-1}<b_{\ell}=0$ with multiplicities $k_{1}, \ldots, k_{\ell}$, and let

$$
\begin{aligned}
& r_{1}=\varrho_{1}+\cdots+\varrho_{k_{1}}, \\
& r_{2}=\varrho_{k_{1}+1}+\cdots+\varrho_{k_{1}+k_{2}}, \ldots, \\
& r_{\ell}=\varrho_{k_{\ell}+1}+\cdots+\varrho_{m} .
\end{aligned}
$$

Then $r_{\ell}=R(\infty)$ and

$$
R(\tau)=\sum_{i=1}^{\ell} \frac{r_{i}}{1-b_{i} \tau}=R(\infty)+\sum_{i=1}^{\ell-1} \frac{r_{i}}{1-b_{i} \tau}
$$


Considering each fraction separately, $R(\tau) \geq R(\infty)+\sum_{i=1}^{\ell} \min \left(0, r_{i}\right)$, so that

$$
R(\infty)+\sum_{i=1}^{\ell} \min \left(0, r_{i}\right) \leq c_{0} \leq R(\infty)
$$

Although crude, this is sometimes sufficient to determine $c_{0}$ exactly. For example, if $\rho_{M}$ is positive semi-definite, then all $r_{i} \geq 0$ and $c_{0}=r_{\ell}=R(\infty) \geq 0$. If in addition $\gamma$ is negative-definite, then $c_{0}=0$. In either case, the total space of $L$ admits a complete, scalar-flat Kähler metric.

In the setting of Theorem $\left[\mathrm{C}\right.$ there is the additional inequality $c_{0}^{\times} \leq R(0)$, necessitated by positivity of the profile (3.7) near $\tau=0$. If the base metric has non-positive Ricci tensor, then $R$ is monotone increasing and $c_{0}^{\times}=R(0)=\sum_{\nu} \varrho_{\nu}$.

3.2. Metrics on Vector Bundles. Let $p:(E, h) \rightarrow\left(D, g_{D}\right)$ be an Hermitian holomorphic vector bundle of rank $n>1$ over a Kähler manifold of dimension $d$. There is a smooth, globally defined norm squared function $r$, just as for line bundles, and the Calabi ansatz has an obvious formulation in this situation, namely to consider closed $(1,1)$-forms

$$
\omega=p^{*} \omega_{D}+\sqrt{-1} \partial \bar{\partial} F(r)=p^{*} \omega_{D}+2 \sqrt{-1} \partial \bar{\partial} f(t) .
$$

The machinery of Theorem $\mathrm{A}$ is easily modified to treat this case. Complexanalytically, the idea is to blow up the zero section of $E$ and pull the form (3.9) back to the total space of the blow-up. The original horizontal data pull back to "partially degenerate" horizontal data since $\omega_{D}$ has rank $d$ as an Hermitian form while the exceptional divisor has dimension $d+n-1$. To avoid problems caused by this degeneracy, the form $\omega_{D}-\gamma$ on $\mathbf{P}\left(E^{\times}\right)$is used as a background metric. The following discussion elaborates on the technicalities necessary to make this idea work.

The tautological bundle, and blowing up. The complement of the zero section of $E$ is denoted by $E^{\times}$, and is regarded either as a complex manifold or as a "punctured $\mathbf{C}^{n}$-bundle" over $D$. There is a free $\mathbf{C}^{\times}$-action on $E^{\times}$by scalar multiplication, whose quotient is the projectivization of $E$, denoted $\mathbf{P}(E)$. The complex manifold $M=\mathbf{P}(E)$ is the total space of a $\mathbf{P}^{n-1}$-bundle $\pi: M \rightarrow D$. The pullback of $E$ to $M$ is the fibre product

$$
\pi^{*} E=\{(\zeta, v) \in M \times E \mid \pi(\zeta)=p(v)\}=M \times_{D} E
$$

endowed with the projection onto the first factor, and the tautological bundle $\tau_{E}$ is the line subbundle of $\pi^{*} E \rightarrow M$ whose fibre at a point $\zeta=(z,[v]) \in \mathbf{P}(E)=M$ is the line through $v \in E_{z}$. Two important observations are:

1. The fibre of $\pi$ over $z \in D$ is the $(n-1)$-dimensional projective space $\mathbf{P}\left(E_{z}^{\times}\right)$, and the restriction of $\tau_{E}$ to a fibre $\mathbf{P}\left(E_{z}^{\times}\right)$is $\mathcal{O}(-1)$. The total space of the latter may be regarded as the blow-up of $\mathbf{C}^{n}$ at the origin.

2. Projection to the second factor in (3.10) induces a biholomorphism $\tau_{E}^{\times} \simeq E^{\times}$, and coincides with the map $\pi: M \rightarrow D$ along the zero section of $\tau_{E}$.

The total space of $\tau_{E}$ is obtained from the total space of $E$ by blowing up the zero section. This is a direct generalization of blowing up a point, and indeed may be regarded as a family of blow-ups of $\mathbf{C}^{n}$, parametrized by points of $D$. 
By abuse of notation, the projection $\tau_{E} \rightarrow M$ is denoted by $p$. These spaces and bundles are organized as follows, with superscripts denoting ranks of bundles or dimensions of spaces:

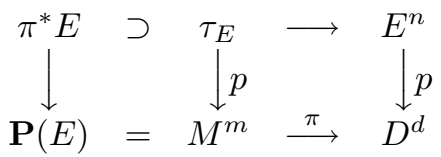

Of course, $n+d=m+1$ since $E^{\times} \simeq \tau_{E}^{\times}$. Via this identification, $L=\tau_{E}$ acquires an Hermitian structure, also denoted $h$, from the Hermitian structure of $E$, and as before $\gamma=\gamma_{1}(L, h)$ denotes the curvature form. The norm squared function $r: E^{\times} \rightarrow(0, \infty)$ may be regarded as a function on $L$, and $p^{*} \gamma=-\sqrt{-1} \partial \bar{\partial} \log r$ as closed $(1,1)$-forms on the total space of $L$. Calabi 4 calls $\gamma$ the "bi-Hermitian curvature form" of $(E, h)$. The preceding discussion makes it clear geometrically that the auxiliary bundle $L$ arises naturally in the Calabi ansatz on vector bundles. Despite the fact that the complex manifolds $L^{\times}$and $E^{\times}$are biholomorphic, it is best to regard them as differential-geometrically distinct; the bundle $E^{\times}$is completed by adding a copy of $D$ (the level set $\{r=0\} \subset E$ ), while $L^{\times}$is completed by adding a copy of $M$ (the level set $\{r=0\} \subset L$ ). Lemma 3.7 below illustrates the importance of this distinction.

Degeneracy in the Calabi ansatz. Let $p:(E, h) \rightarrow\left(D, g_{D}\right)$ be an Hermitian holomorphic vector bundle of rank $n$ over a Kähler manifold. The closed $(1,1)$-form $\pi^{*} \omega_{D}$ may be denoted by $\omega_{D}$ for brevity, as in equation (3.11) below. Associated to a profile $\varphi$ are functions $\mu$ and $f$ as in Section 2. Upon blowing up the zero section of $E$, the closed $(1,1)$-form $\omega=p^{*} \omega_{D}+2 \sqrt{-1} \partial \bar{\partial} f(t)$ is immediately written, using results of Section 2, as

$$
\omega=\varphi(\tau)\left(d t \wedge d^{c} t\right)+p^{*} \omega_{D}-\tau p^{*} \gamma
$$

The form $\pi^{*} \omega_{D}$ on $M$ is degenerate along the fibres of $\pi: M \rightarrow D$, while under the hypotheses of Theorem $\mathrm{D}$ the form $\pi^{*} \omega_{D}-\gamma$ is a Kähler form on $M$, and (by Lemma 3.6 below) the horizontal data $p:\left(\tau_{E}, h\right) \rightarrow\left(M, \omega_{M}\right)$ are $\sigma$-constant. The vector bundle data $p:(E, h) \rightarrow\left(D, \omega_{D}\right)$ are said to be $\sigma$-constant in this situation. Before defining the analogues of the functions $Q$ and $R$ for vector bundle data, it is reassuring to verify that the choice of background metric in the family $\omega_{M}(\tau)$ is immaterial in the following sense.

Lemma 3.6. If $\omega_{M}(\tau)$ is a Kähler form for every $\tau>0$, and if $p:(L, h) \rightarrow$ $\left(M, \omega_{M}\left(\tau_{0}\right)\right)$ is $\sigma$-constant for some $\tau_{0}>0$, then $p:(L, h) \rightarrow\left(M, \omega_{M}(\tau)\right)$ is $\sigma$-constant for every $\tau>0$.

Proof. Fix $\tau_{0}>0$, and regard $\omega_{M}\left(\tau_{0}\right)$ as a background metric, so that all index raising and lowering is done with respect to this metric. Let $\mathrm{B}$ denote the curvature endomorphism of $(L, h)$; by assumption, the eigenvalues $\beta_{\nu}$ are constant on $M$, and the trace of the Ricci form $\rho_{M}\left(\tau_{0}\right)$ with respect to the Kähler form $\omega_{M}(\tau)$ is constant for all $\tau>0$. Consider the family $A(\tau)$ of endomorphisms associated to the closed $(1,1)$-forms $\omega_{M}(\tau)$. Then $A\left(\tau_{0}\right)$ is the identity, and $A^{\prime}(\tau)=-\mathrm{B}$, so

$$
A(\tau)=\mathrm{I}-\left(\tau-\tau_{0}\right) \mathrm{B} \quad \text { for } \tau \geq 0 .
$$

The eigenvalues of $\mathrm{B}$ are $\beta_{1}, \ldots, \beta_{d}$, and $-1 / \tau_{0}$ (the latter of multiplicity $n-1$ ). Thus $A(\tau)$ has constant eigenvalues for each $\tau \geq 0, A(0)=\mathrm{I}+\tau_{0} \mathrm{~B}$ has rank $d$, and 
the endomorphisms $A(\tau)$ have the same eigenbundles. Set

$$
Q\left(\tau, \tau_{0}\right)=\operatorname{det} A(\tau)=\frac{\tau^{n-1}}{\tau_{0}^{n-1}} \prod_{\nu=1}^{d}\left(1-\left(\tau-\tau_{0}\right) \beta_{\nu}\right)
$$

so that for each $\tau_{0}>0, Q$ is a constant-coefficient polynomial with a zero of order $(n-1)$ at $\tau=0$. Then $Q\left(\tau, \tau_{0}\right)$ is the ratio of the volume forms of $\omega_{M}(\tau)$ and $\omega_{M}\left(\tau_{0}\right)$, and is constant on $M$ for each fixed $\tau>0$, so the Ricci forms $\rho_{M}(\tau)$ and $\rho_{M}\left(\tau_{0}\right)$ coincide. Consequently, if the trace of the Ricci form $\rho_{M}\left(\tau_{0}\right)$ with respect to $\omega_{M}(\tau)$ is constant for all $\tau>0$, then the same is true of the Ricci form $\rho_{M}\left(\tau_{1}\right)$ for every $\tau_{1}>0$.

Suppose $p:(E, h) \rightarrow\left(D, \omega_{D}\right)$ are $\sigma$-constant data, and set $\omega_{M}(\tau)=\pi^{*} \omega_{D}-\tau \gamma$. The background metric on $M$ is taken to be $\omega_{M}:=\omega_{M}(1)$, in contrast to the notation used for line bundles. As noted in the proof of Lemma 3.6] the Ricci forms $\rho_{M}(\tau)$ do not depend on $\tau$, and are denoted simply by $\rho_{M}$. By contrast, the Ricci endomorphisms, defined by $\varrho_{M}(\tau):=\omega_{M}(\tau)^{-1} \rho_{M}$, do depend on $\tau$, though by hypothesis they all have constant trace. More is true: For each $\tau>0$, the vertical tangent bundle of $M$ (namely, ker $\pi_{*}$ ) is an eigenbundle of $\varrho_{M}(\tau)$ with eigenvalue $n$ (of multiplicity $n-1$ ). To see this, observe that the global inner product $\left\langle\rho_{M}, \gamma\right\rangle$ is constant on $M$ since $\left\langle\rho_{M}, \omega_{M}(\tau)\right\rangle$ is constant on $M$ for each $\tau>0$. On a fibre of $\pi$, which is a projective space, $-\gamma$ is a Kähler form, and since the trace of $\rho_{M}$ restricted to the fibre is constant, the restriction must be a multiple of $-\gamma$, which implies the Ricci endomorphism is a multiple of the identity on each fibre. For cohomological reasons, the eigenvalue is $n$. by

The functions $Q$ and $R$ are defined with respect to the background metric $\omega_{M}$

$$
\begin{aligned}
& Q(\tau)=\operatorname{det} A(\tau)=\tau^{n-1} \prod_{\nu=1}^{d}\left(1+\beta_{\nu}-\tau \beta_{\nu}\right)=: \tau^{n-1} Q_{0}(\tau), \\
& R(\tau)=\operatorname{tr}\left(A(\tau)^{-1} \varrho\right)=\frac{n(n-1)}{\tau}+\text { smooth. }
\end{aligned}
$$

Note that $Q$ has a zero of order $(n-1)$ and $R$ has a simple pole at $\tau=0$.

Proof of Theorem D. As in Section 2, a profile $\varphi$ induces a bundle-adapted metric on $E$ whose scalar curvature is

$$
\sigma_{\varphi}(\tau)=\left(R-\frac{1}{2 Q}(\varphi Q)^{\prime \prime}\right)(\tau)
$$

The function $\sigma_{\varphi}$ generally has a pole at $\tau=0$. Interestingly, the scalar curvature is bounded near the zero section if, and only if, the metric extends over the zero section:

Lemma 3.7. If the metric (3.9) is complete, then the function $\sigma_{\varphi}$ in equation (3.13) has a removable singularity at $\tau=0$ if and only if the profile satisfies the boundary conditions

$$
\varphi(0)=0, \quad \varphi^{\prime}(0)=2,
$$

if and only if the metric extends over the zero section of $E$. 
Proof. Completeness of the metric dictates that $\varphi(0)=0$. Writing $\varphi(\tau)=\varphi^{\prime}(0) \tau+$ $O\left(\tau^{2}\right)$ near $\tau=0$ - so that $(\varphi Q)(\tau)=\varphi^{\prime}(0) \tau^{n} Q_{0}(\tau)+O\left(\tau^{n+1}\right)$ - and differentiating twice gives

$$
\sigma_{\varphi}(\tau)=\frac{n(n-1)}{\tau}\left(1-\frac{\varphi^{\prime}(0)}{2}\right)+O(1)
$$

so the singularity at $\tau=0$ is removable if and only if $\varphi^{\prime}(0)=2$. The remaining assertions are proven exactly as in the line bundle case.

Lemma 3.7 says the Calabi ansatz (3.9) does not give rise to a complete Kähler metric of constant scalar curvature on the punctured disk subbundle of $E$. Of course, the punctured disk subbundle of $E$ is biholomorphic to the punctured disk subbundle of the line bundle $\tau_{E}$; the difference between Theorem Cland the present situation is that here the forms $\omega_{M}(\tau)$ drop in rank at $\tau=0$. As a specific example, on the punctured projective space $\mathbf{P}^{m} \backslash\{\mathrm{pt}\}$ (with $m \geq 2$ ) there does not exist a complete Kähler metric whose scalar curvature is bounded below near the puncture, cf. Calabi (4], p. 276).

The differential equation obtained by setting (3.13) equal to $c$ has a regular singular point at $\tau=0$, and the general solution is

$$
\varphi(\tau)=\frac{2}{Q(\tau)}\left(\alpha_{0}+\alpha_{1} \tau+\int_{0}^{\tau}(R(x)-c)(\tau-x) Q(x) d x\right) .
$$

Because $Q$ has a zero of order $n-1$ at $\tau=0, \varphi(0)=0$ forces $\alpha_{0}=\alpha_{1}=0$, so the purported profile for a bundle-adapted metric of scalar curvature $c$ on the disk subbundle of $E$ is

$$
\varphi(\tau)=\frac{2}{Q(\tau)} \int_{0}^{\tau}(R(x)-c)(\tau-x) Q(x) d x .
$$

This profile satisfies the boundary conditions $\varphi(0)=0$ and $\varphi^{\prime}(0)=2$. The first results from two applications of l'Hôpital's rule. To obtain the latter, differentiate $\varphi Q$ and solve for $\varphi^{\prime}$ to get

$$
\varphi^{\prime}(\tau)=-\varphi(\tau) \frac{Q^{\prime}}{Q}(\tau)+\frac{2}{Q} \int_{0}^{\tau}(R(x)-c) Q(x) d x .
$$

Writing $\varphi(\tau)=\varphi^{\prime}(0) \tau+O\left(\tau^{2}\right)$ and using l'Hôpital's rule gives

$$
\begin{aligned}
\varphi^{\prime}(0) & =\lim _{\tau \rightarrow 0}\left(-\varphi^{\prime}(0)(n-1)+O(\tau)+2 \frac{(R(x)-c) Q(\tau)}{Q^{\prime}(\tau)}\right) \\
& =-\varphi^{\prime}(0)(n-1)+2 n,
\end{aligned}
$$

so $\varphi^{\prime}(0)=2$ as claimed. Most of the remaining points are checked exactly as in the proof of Theorem B, using the profile (3.14). The Einstein condition $\rho_{\varphi}=\lambda \omega_{\varphi}$ is equivalent to

$$
\lambda \pi^{*} \omega_{D}=\rho_{M}+n \gamma \quad \text { and } \quad \frac{1}{2 Q}(\varphi Q)^{\prime}(\tau)=n-\lambda \tau .
$$

\section{Limitations, Examples, and Literature Survey}

The chief issue addressed in this section is the extent to which the hypothesis of $\sigma$-constancy is necessary for the Calabi ansatz to yield metrics of constant scalar curvature. In addition, we give detailed examples of data satisfying the hypotheses of the existence theorems proven in Section 3. prove a few miscellaneous technical 
results, and briefly survey the existing literature, particularly contrasting the three main approaches used by prior authors.

4.1. Scope of the Momentum Construction. This subsection describes our attempt to understand the limitations of the Calabi ansatz. The general construction begins with horizontal data - an Hermitian holomorphic line bundle over a complete Kähler manifold, and an interval of real numbers - and associates to each profile $\varphi$ a Kähler metric $g_{\varphi}$. The role of $\sigma$-constancy in reducing a PDE to an ODE is discussed below (Level sets of the scalar curvature); these remarks are intended to motivate the proof of Theorem $\mathrm{E}$

Normalization of the momentum interval. There are two intervals in the Calabi ansatz: The momentum interval $I$, which is closely related to the area of the fibre metric; and the $r$ interval defining the $S^{1}$-invariant domain on which the metric is defined. That these intervals are essentially independent is clear from Table 2.1.

Lemma 4.1 below justifies the assumption inf $I=0$ made in our existence theorems. Begin with horizontal data $\left\{p:(L, h) \rightarrow\left(M, g_{M}\right), I\right\}$. The horizontal forms $\omega_{M}-\tau \gamma$ are assumed to be positive and complete for all $\tau \in I$, so if $I=\mathbf{R}$, then $\gamma=0$, and every profile gives rise to a local product metric. In all other cases, $I$ may be normalized without loss of generality; in words, every bundle-adapted metric is isometric to a bundle-adapted metric having momentum interval $I$ equal to $\mathbf{R}$, or else having $\inf I=0$ :

Lemma 4.1. If $\left\{p^{\prime}:\left(L^{\prime}, h^{\prime}\right) \rightarrow\left(M, g_{M}^{\prime}\right), I^{\prime}\right\}$ are horizontal data with $I^{\prime} \neq \mathbf{R}$ and if $\psi$ is a profile on $I^{\prime}$ inducing a metric $g_{\psi}$, then there exist horizontal data $\left\{p:(L, h) \rightarrow\left(M, g_{M}\right), I\right\}$, with inf $I=0$, and a profile $\varphi$ on $I$ such that $g_{\varphi}$ and $g_{\psi}$ are isometric.

Proof. Suppose first that $I^{\prime}$ is bounded below, and set $a=\inf I^{\prime}$; thus $\omega_{M}^{\prime}-a \gamma^{\prime}$ is a Kähler form on $M$. The 'translated' data

$$
(L, h)=\left(L^{\prime}, h^{\prime}\right), \quad \omega_{M}=\omega_{M}^{\prime}-a \gamma^{\prime}, \quad I=I^{\prime}-a, \quad \varphi(\tau)=\psi(\tau-a),
$$

have the advertised properties.

Now suppose $I^{\prime} \neq \mathbf{R}$ is not bounded below. By a translation argument analogous to that just given, it may be assumed that $\sup I^{\prime}=0$. Consider the 'inverted' data

$$
(L, h)=\left(L^{\prime *}, h^{-1}\right), \quad \omega_{M}=\omega_{M}^{\prime}, \quad I=-I^{\prime}, \quad \varphi(\tau)=\psi(-\tau) .
$$

The $t$ integral acquires a sign change, which corresponds to the inversion map $\iota:(L, h) \rightarrow\left(L^{*}, h^{-1}\right)$, given locally by $z^{0} \mapsto 1 / z^{0}$ in a line bundle chart. It is straightforward to verify $g_{\varphi}=\iota^{*} g_{\psi}$.

The role of $\sigma$-constancy in the Calabi ansatz. According to Theorem $\mathrm{A}$ and equation (2.12), the scalar curvature $\sigma_{\varphi}$ is given in terms of horizontal data and the profile by

$$
\sigma_{\varphi}=\sigma_{M}(\tau)-\frac{1}{2 Q} \frac{\partial^{2}}{\partial \tau^{2}}(Q \varphi)(\tau), \quad \sigma_{M}(\tau)=R(\tau)-\square_{\omega_{M}(\tau)} \log Q
$$

Our aim has been to find data (bundle, metrics, interval, and profile) such that $\sigma_{\varphi}$ is constant. We expect, however, that for "generic" horizontal data the level sets of $\sigma_{\varphi}$ do not coincide with the level sets of $\tau$, regardless of the choice of profile. Consequently, the Calabi ansatz "usually" does not yield a metric of constant scalar 
curvature. It is natural to seek geometric conditions on the horizontal data that are necessary and sufficient for existence of a profile inducing a metric of constant scalar curvature (or even having scalar curvature depending only upon $\tau$ ). The condition of $\sigma$-constancy is sufficient, by the existence theorems of the preceding section. Theorem E, to which we now turn, gives good partial information about necessity.

Proof of Theorem E, and related remarks. Equation (4.1) holds for all profiles and horizontal data, and it is reasonable to regard specification of the scalar curvature as a family of ODEs on $I$, parametrized by points of $M$. Assume $\sigma$ is a (smooth) function on $I$. The equation $\sigma_{\varphi}=\sigma(\tau)$ is trivially integrated to

$$
\begin{aligned}
\varphi(\tau, z)=\frac{1}{Q(\tau, z)}[\varphi(0, z)+ & \frac{\partial \varphi}{\partial \tau}(0, z) \tau \\
& \left.+2 \int_{0}^{\tau}(\tau-x)\left(\sigma_{M}(x, z)-\sigma(x)\right) Q(x, z) d x\right] .
\end{aligned}
$$

The solution is viewed as a function $\varphi: I \times M \rightarrow \mathbf{R}$, and the general problem is to determine when this function depends only on $\tau \in I$. Specifically, the intent is to assume the solution depends only on $\tau$ for some choice(s) of $\sigma$, and to deduce that the horizontal data are $\sigma$-constant. For obvious reasons, it is desirable to make minimal assumptions on $\sigma$. The arguments below require real-analyticity; this is adequate to deduce interesting geometric consequences. (More accurately, Theorem E assumes existence of two profiles inducing metrics with real-analytic scalar curvature functions. The latter need not be distinct. Further partial results give some consequences of existence of a single profile.)

Fix horizontal data $\left\{p:(L, h) \rightarrow\left(M, g_{M}\right), I\right\}$. Suppose there exist real-analytic functions $\sigma_{1}$ and $\sigma_{2}$ on $I$ so that the corresponding profiles $\varphi_{1}$ and $\varphi_{2}$ given by (4.2) depend only on $\tau$ and agree at $\tau=0$. Then the function

$$
\begin{aligned}
\psi(\tau):=\left(\varphi_{2}-\varphi_{1}\right)(\tau)=\frac{1}{Q(\tau, z)}\left[\left(\varphi_{2}^{\prime}(0)\right.\right. & \left.-\varphi_{1}^{\prime}(0)\right) \tau \\
& \left.+\int_{0}^{\tau}(\tau-x)\left(\sigma_{1}(x)-\sigma_{2}(x)\right) Q(x, z) d x\right]
\end{aligned}
$$

depends only on $\tau$ and vanishes at 0 . Let $N(\tau, z)$ denote the term in square brackets. The aim is to show that the horizontal data are $\sigma$-constant, namely that $Q(\tau, z)$ depends only on $\tau$ and the horizontal metric $g_{M}(\tau)$ has constant scalar curvature for each $\tau \in I$. Because $\sigma_{\varphi}$ is assumed to depend only upon $\tau$ (for $\varphi=\varphi_{1}$, say), it suffices to show that $Q$ depends only on $\tau$, since $\sigma_{M}(\tau)=\sigma_{\varphi}+(1 / 2 Q)(\varphi Q)^{\prime \prime}(\tau)$. To this end, it is enough to show that $N$ depends only on $\tau$ since

$$
N^{\prime \prime}(\tau, z)=\left(\sigma_{1}(\tau)-\sigma_{2}(\tau)\right) Q(\tau, z)
$$

The function $\psi$ is real-analytic and not identically zero (since $\varphi_{1}$ and $\varphi_{2}$ are distinct). Put $\alpha=\varphi_{2}^{\prime}(0)-\varphi_{1}^{\prime}(0)$, write $\sigma_{1}(\tau)-\sigma_{2}(\tau)=S(\tau)$, and consider the initial-value problem

$$
y^{\prime \prime}(\tau)-\frac{S(\tau)}{\psi(\tau)} y(\tau)=0, \quad y(0)=0, \quad y^{\prime}(0)=\alpha .
$$

For each $z \in M$, the function $y=N(, z)$ satisfies the singular initial-value problem (4.3). Because the coefficients depend only on $\tau$ (and the initial values are 
constant), an easy power series calculation shows that $N$ is independent of $z$. This completes the proof Theorem E

Even the case $\sigma_{1}=\sigma_{2}=c$ is of geometric interest: If $\{p, I\}$ are horizontal data, and there exists a fibre-complete, bundle-adapted metric of scalar curvature $c$ in $\Delta(L)$ (so that $\varphi_{1}^{\prime}(0)=2$ ) and a similar metric in $\Delta^{\times}(L)\left(\right.$ so $\left.\varphi_{2}^{\prime}(0)=0\right)$, then the data are $\sigma$-constant; compare Theorems B and $\mathrm{C}$.

Theorem E is of course local; nothing is assumed about completeness of the base or fibre metric. The key hypothesis is existence of two profiles whose scalar curvature is independent of $\tau$; taking the difference $\varphi_{2}-\varphi_{1}$ cancels the relatively complicated (but profile-independent) term $\sigma_{M}(\tau)$.

By Theorem E, it is reasonable to assert that the Calabi ansatz is exhausted, so far as families of Kähler metrics of constant scalar curvature are concerned: If $\{p, I\}$ is not $\sigma$-constant, then there is at most one metric (total) of constant scalar curvature that arises from the Calabi ansatz in the disk bundle $\Delta(L)$, the punctured disk bundle $\Delta^{\times}(L)$, or in a partial contraction of the zero section. We conclude this section with a few remarks on the consequences of assuming existence of a single profile whose induced metric has constant scalar curvature. These calculations are included mostly for (pedagogical) completeness.

Problem 4.2. Let $\{p, I\}$ be horizontal data, and suppose there exists a profile $\varphi$ depending only on $\tau$ such that $\sigma_{\varphi}$ is constant. Are the horizontal data necessarily $\sigma$-constant?

We are not presently able to answer this question, though we believe the answer is "yes." While falling short of a proof, our calculations provide evidence and yield some suggestive partial results.

The approach is to draw conclusions about the curvature of horizontal data under the assumption that there exists a germ of a profile at $\tau=0$, satisfying $\varphi(0)=0$ and $\varphi^{\prime}(0)=2$, and inducing a bundle-adapted metric whose scalar curvature depends only on $\tau$. This investigation only involves consideration of rational functions defined on $I \times U$, with $U$ a neighbourhood of the origin in $\mathbf{C}^{m}$. Solutions of ODEs defining momentum profiles are considered in the 'non-geometric regime' where $\tau<0$. Nothing is being asserted about bundle-adapted Kähler metrics for $\tau<0$, of course; what is being used is real-analyticity of $\varphi$ in $\tau$, and the fact that if such a function $\varphi(\tau, z)$ depends only on $\tau$ for $\tau \geq 0$, then the same is true for $\tau<0$.

Proposition 4.3. Fix horizontal data $\left\{p:(L, h) \rightarrow\left(M, g_{M}\right)\right.$, I $\}$ and a polynomial function $\sigma$. If there exists a profile $\varphi(\tau)$ such that $\sigma_{\varphi}=\sigma(\tau)$, then $\varphi$ is a rational function.

Proof. Fix $z \in M$, and let $\varphi(\tau, z)$ be the function defined by (4.2). It will be shown that a non-rational term in $\varphi$ has a logarithmic singularity at a root of $Q$, and that this root must depend on $z$, so $\varphi$ depends on $z$ as well. These logarithmic terms potentially arise from $\square_{\omega_{M}(\tau)} \log Q$, which enters via $\sigma_{M}(\tau)=R(\tau)-\square_{\omega_{M}(\tau)} \log Q$.

It is enough to work in a coordinate neighbourhood $U$ around $z \in M$. Let $\left\{\beta_{\nu}(z)\right\}_{\nu=1}^{m}$ denote the (a priori non-constant) eigenvalues of the curvature endomorphism $\mathrm{B}$ in $U$, and let $b_{1}<\cdots<b_{\ell} \leq 0$ be the distinct eigenvalues, of multiplicity $k_{i}$; without loss of generality, $k_{i}$ may be assumed constant throughout $U$. 
Then

$$
Q(\tau, z)=\prod_{\nu=1}^{m}\left(1-\tau \beta_{\nu}(z)\right)=\prod_{i=1}^{\ell}\left(1-\tau b_{i}(z)\right)^{k_{i}}
$$

so

$$
\log Q(\tau, z)=\sum_{i=1}^{\ell} k_{i} \log \left(1-\tau b_{i}(z)\right) .
$$

Choose a unitary eigenframe $\left\{\mathbf{e}_{\nu}\right\}_{\nu=1}^{m}$ in $U$, and let $\left(z^{\nu}\right)$ be complex local coordinates such that for $1 \leq \nu \leq m, z^{\nu}=0$ and $\partial_{\nu}=\mathbf{e}_{\nu}$ at $z$. Differentiating $\log Q(\tau, z)$, using subscripts (after a comma) to denote partial derivatives, gives

$$
\partial_{\lambda} \bar{\partial}_{\mu} \log Q=\sum_{i=1}^{\ell}\left(\frac{k_{i} \tau^{2} b_{i, \lambda} b_{i, \bar{\mu}}}{\left(1-\tau b_{i}\right)^{2}}-\frac{k_{i} \tau b_{i, \lambda \bar{\mu}}}{\left(1-\tau b_{i}\right)}\right) .
$$

Let $\left\{\theta_{\nu}\right\}$ be the basis of $\bigwedge_{\mathbf{0}}^{(1,0)}$ dual to $\left\{\mathbf{e}_{\nu}\right\}$, so along the fibre at $\mathbf{0}$ the metric and its inverse are given by

$$
\omega_{M}(\tau)=\sum_{\nu=1}^{m}\left(1-\beta_{\nu} \tau\right) \theta_{\nu} \bar{\theta}_{\nu}, \quad \omega_{M}(\tau)^{-1}=\sum_{\nu=1}^{m} \frac{e_{\nu} \bar{e}_{\nu}}{\left(1-\beta_{\nu} \tau\right)} .
$$

Taking the trace of (4.4) with respect to $\omega_{M}(\tau)^{-1}$, and using the fact that $\left\{\mathbf{e}_{\nu}\right\}$ is a coordinate basis at $\mathbf{0}$,

$$
\begin{aligned}
& \square_{\omega_{M}(\tau)} \log Q(\tau, z) \\
& \quad=\sum_{i=1}^{\ell} \sum_{\lambda=1}^{m}\left(\frac{k_{i} \tau^{2}\left|b_{i, \lambda}\right|^{2}}{\left(1-\tau \beta_{\lambda}\right)\left(1-\tau b_{i}\right)^{2}}-\frac{k_{i} \tau b_{i, \lambda \lambda}}{\left(1-\tau \beta_{\lambda}\right)\left(1-\tau b_{i}\right)}\right)
\end{aligned}
$$

at $\mathbf{0}$. When the right-hand side is expanded in partial fractions, the coefficients of the principal part at $\tau=1 / b_{i}$ are linear combinations of derivatives of $b_{i}$. In particular, if a principal part appears, then $b_{i}$ is non-constant.

The integrand in (4.2) is rational for each $z \in M$, and the only non-polynomial terms come from (4.5). In other words, $\varphi(\tau, z)$ is the integral of a rational function, and any non-rational (logarithmic) terms arise from a principal part. But as just observed, a principal part is non-vanishing only when some $b_{i}$ is non-constant, and this implies $\varphi$ depends on $z$.

Under similar hypotheses, the calculations in the proof can be used to read off other geometrically interesting consequences. When the curvature endomorphism B is a multiple of the identity, examination of the highest-order pole in (4.5) implies $\sigma$-constancy:

Proposition 4.4. Let $\{p, I\}$ be horizontal data such that $B(z)=\beta(z) \mathrm{I}$ for some smooth function $\beta$. Assume there is a profile $\varphi(\tau)$ inducing a metric whose scalar curvature is $\sigma(\tau)$ for some polynomial $\sigma$. Then the data are $\sigma$-constant.

Finally, the non-constant eigenvalues of the curvature are tightly constrained by the assumptions made above.

Proposition 4.5. Let $\{p, I\}$ be horizontal data, and let $\left\{b_{i}(z)\right\}_{i=1}^{\ell}$ denote the distinct eigenvalues of the curvature endomorphism. Suppose there exists a profile, depending only on $\tau$ and inducing a metric whose scalar curvature depends only 
on $\tau$. If $b_{i}(z)$ is non-constant, then for every $z \in M,\left(1-\tau b_{i}\right)^{k_{i}}$ divides the term in square brackets in (4.2).

Proof. By hypothesis, $\varphi$ - and in particular each pole - is independent of $z$. So if $\left(1-\tau b_{i}\right)^{k_{i}}$ does not divide the numerator (the term in square brackets) in (4.2), then $b_{i}$ is independent of $z$.

The condition that the numerator should have such a factor is a strong constraint on the way in which $b_{i}(z)$ can vary. Unfortunately, it is a constraint whose consequences seem hard to express in simple fashion.

4.2. Line Bundles. This section describes some examples of data satisfying the hypotheses of Theorems B and C. The "atomic" examples are suitable line bundles over Hodge manifolds of constant scalar curvature; even when the base is a curve, interesting metrics arise.

Scalar-flat metrics on complex surfaces. It is instructive to see what emerges from the momentum construction when the (complex) dimension of the base $M$ is 1. (The case $\operatorname{dim} M=0$ was dealt with in Section 2.2 see Table 2.1.)

The conditions of $\sigma$-constancy are satisfied if and only if $\rho_{M}=\lambda \omega_{M}$ and $\gamma=$ $\beta \omega_{M}$, for constants $\lambda$ and $\beta$. Then $Q(\tau)=1-\beta \tau$ and $R(\tau)=\lambda(1-\beta \tau)^{-1}$. The profiles with $\varphi(0)=0$ which yield a metric of constant scalar curvature $c$ are given, with their derivative at 0 , by

$$
\varphi(\tau)=\frac{2 \tau+(\lambda-c) \tau^{2}+c \beta \tau^{3} / 3}{1-\beta \tau}, \quad \varphi^{\prime}(0)=2
$$

or

$$
\varphi(\tau)=\frac{(\lambda-c) \tau^{2}+c \beta \tau^{3} / 3}{1-\beta \tau}, \quad \varphi^{\prime}(0)=0 .
$$

It is easy to analyze the choices of $\beta, \lambda$, and $c$ that give rise to positive profiles and hence to complete metrics. However, the most interesting case is that where $c=0$, for then the metric is anti-self-dual in the sense of 4-dimensional conformal geometry. In addition to recovering a number of known examples, we find new metrics on $\mathbf{C}^{2}$ and suitable quotients by $\mathbf{Z}$ and $\mathbf{Z} \oplus \mathbf{Z}$.

The first case to consider is when $\lambda>0$, so $M=\mathbf{P}^{1}$ with a Fubini-Study (round) metric, normalized so that $\lambda=1$. In this case, if $L=\mathcal{O}(-k)$, then $\beta=-k / 2$; the factor of 2 arises because the canonical bundle is $\mathcal{O}(-2)$. Equation 4.6 takes the form

$$
\varphi_{k}(\tau)=\frac{2 \tau+\tau^{2}}{1+k \tau / 2}
$$

which is clearly positive on $(0, \infty)$ if $k \geq 0$. The growth is linear at $\infty$, so the corresponding metric $\omega_{k}$ lives on the line bundle (not some disk subbundle).

By Theorem B, $\omega_{k}$ is Einstein if and only if $k=2$. The resulting metric - the Eguchi-Hanson graviton - is an example of the Ricci-flat metric on $T^{*} \mathbf{P}^{d}$ found by Calabi [4. If $k=1$ the metric is the Burns metric on the blow-up of $\mathbf{C}^{2}$ at the origin [18] 19]. If $k \geq 3$ the metrics are those found by LeBrun in [18].

In the case $\lambda<0$, equation (4.6) never yields complete metrics; the corresponding profiles are all negative for large $\tau$, but if $b$ is the first positive zero then $\varphi^{\prime}(b)=-2$ if and only if $\beta=0$. This case does, however, yield compact extremal Kähler metrics, see [35]. 
Finally, if $\lambda=0$ - so the universal cover of $M$ is $\mathbf{C}$ - then (4.6) reduces to

$$
\varphi(\tau)=\frac{2 \tau}{1-\beta \tau} .
$$

This profile yields a complete metric on $L=\mathcal{O}_{\mathbf{C}}$, whose total space is $\mathbf{C}^{2}$, provided $\beta<0$. These complete, scalar-flat Kähler metrics on $\mathbf{C}^{2}$ are not Einstein, and appear to be new. The formula $h_{\beta}:=\exp \left(\beta(\operatorname{Imz})^{2}\right)$ defines an Hermitian metric of constant, negative curvature; indeed, $\gamma\left(\mathcal{O}_{\mathbf{C}}, h_{\beta}\right)=\beta \omega$, where $\omega=(\sqrt{-1} / 2) d z \wedge d \bar{z}$ is the standard Kähler form.

If the Hermitian metric $h$ is suitably translation-invariant, then the profile (4.8) gives rise to complete metrics of zero scalar curvature on $(\mathbf{C} / \mathbf{Z}) \times \mathbf{C}$ and on the total space of a line bundle of negative degree over an elliptic curve. If $\beta$ is integral, $h_{\beta}$ is an example of such a $\mathbf{Z} \oplus \mathbf{Z}$-invariant metric.

Bundles over product manifolds. The basic example of $\sigma$-constant horizontal data comes from a combination of some well-known results:

Lemma 4.6. Let $(M, g)$ be a Hodge manifold of constant scalar curvature. Then there exists an Hermitian line bundle $p:(L, h) \rightarrow(M, g)$ such that the data $\{p,[0, \infty)\}$ are $\sigma$-constant.

Proof. By the Lefschetz Theorem on $(1,1)$-classes, there exists a holomorphic line bundle $L$ whose first Chern class is $[-\omega]$. To see there is an Hermitian structure $h$ with $\gamma(L, h)=-2 \pi \omega$, start with an arbitrary Hermitian structure $h_{0}$ and let $\gamma_{0}$ be the curvature form. By the Hodge Theorem, there exists a unique smooth, real-valued function $u$ satisfying

$$
\gamma-\gamma_{0}=\sqrt{-1} \partial \bar{\partial} u, \quad \int_{M} u \operatorname{dvol}_{g}=0 .
$$

Put $h=e^{-u} h_{0}$. For this Hermitian structure, the horizontal forms $\omega_{M}(\tau)$ are positive -indeed, are homothetic to $\omega_{M}$ - hence have constant scalar curvature for all $\tau \geq 0$. Finally, the curvature endomorphism is a scalar multiple of the identity, in particular has constant eigenvalues.

There are trivial improvements on the statement; the Kähler form need only be homothetic to an integral form, and every positive power of $L$ admits a suitable Hermitian structure. It is also clear that there are examples of $\sigma$-constant horizontal data over certain non-compact manifolds, such as Hermitian symmetric spaces.

Remark 4.7. It is tempting to 'iterate' the momentum construction, using a constant scalar curvature metric $g_{\varphi}$ on a disk bundle $\Delta(L)$ as the base metric for appropriate horizontal data. While this is sometimes possible, it is noteworthy that the 'natural' choice of line bundle $-L$ pulled back over its own total space, equipped with the induced Hermitian structure - does not fit into this framework. Indeed, if $(L, h)$ is non-flat, then the curvature of the pullback bundle, computed with respect to an arbitrary bundle-adapted metric on the disk bundle, does not have constant eigenvalues.

For data as in Lemma 4.6, the functions $P, Q$, and $R$ are written explicitly as follows: Let the constant scalar curvature of the Hodge manifold $\left(M^{m}, g\right)$ be $\sigma$, and let $p:(L, h) \rightarrow(M, g)$ be the Hermitian line bundle with curvature $\gamma=-2 \pi \omega$. For each positive integer $k$, the bundle $\left(L^{k}, h^{k}\right)$ has curvature $k \gamma$. Fix $\alpha>0$, and equip 
$M$ with the metric $g_{M}=2 \pi \alpha g$. Together with the compatible interval $[0, \infty)$, these data are $\sigma$-constant, and

$$
\begin{aligned}
& Q(\tau)=\left(1-\frac{k}{\alpha} \tau\right)^{m}, \quad R(\tau)=\frac{\alpha \sigma}{\alpha-k \tau}, \\
& P(\tau)=2 \sigma\left(1-\frac{k}{\alpha} \tau\right)^{m-1} .
\end{aligned}
$$

Since every positive or negative Einstein-Kähler metric is (homothetic to) a Hodge metric, Lemma 4.6 encompasses most prior examples of horizontal data to which Theorems $\mathrm{B}$ and $\mathrm{C}$ may be applied. It is reasonable to ask whether or not a Hodge metric of constant scalar curvature is "essentially" Einstein-Kähler. The answer is "no," even discounting product metrics, homogeneous metrics, and the like. LeBrun 20 constructed scalar-flat metrics on certain blown-up ruled surfaces; the Kähler classes of these metrics depend on a real parameter, and the class is rational when the parameter value is rational. Higher-dimensional examples include the manifolds obtained from $\mathbf{P}^{2 k+1}$ by blowing up a pair of skew $\mathbf{P}^{k}$ 's. Such a manifold is a $\mathbf{P}^{1}$-bundle over $\mathbf{P}^{k} \times \mathbf{P}^{k}$, and a Kähler class is determined by the area of a $\mathbf{P}^{1}$ fibre and by the areas of lines in each of the base factors. If the latter are equal, and are a rational multiple of the area of a fibre, then the Kähler class is proportional to a Hodge class, and by [12] (Remark 5.3, p. 584) is represented by a metric of constant scalar curvature.

The family $g_{M}(\tau)$ of horizontal metrics arising from $\sigma$-constant horizontal data need not be homothetic. A simple way to arrange this is to take a suitable product of data arising from Lemma 4.6 .

Lemma 4.8. Let $\left\{p_{j}:\left(L_{j}, h_{j}\right) \rightarrow\left(M_{j}, g_{j}\right),[0, \infty)\right\}, j=1, \ldots, n$, be $\sigma$-constant horizontal data. Then the line bundle

$$
p: L=\bigotimes_{j=1}^{n} \pi_{j}^{*} L_{j} \longrightarrow M=M_{1} \times \cdots \times M_{n},
$$

$\pi_{j}: M \rightarrow M_{j}$ the projection, equipped with the induced metrics, is $\sigma$-constant.

The proof is immediate. The corresponding function $Q$ is a product of terms as in equation (4.9), while $R$ is a sum of such terms.

If $\left(M_{j}, g_{j}\right)$ are Einstein-Kähler, then the construction just described gives rise to complete metrics on tensor products of pluri-canonical and pluri-anticanonical bundles as in Corollaries B.2 and B.3.

Remarks about compact Einstein-Kähler metrics. By Yau and Aubin's solution of the Calabi conjecture, simply-connected Einstein-Kähler manifolds of nonpositive scalar curvature are plentiful, the simplest examples being smooth complete intersection varieties of degree $k \geq N+1$ and dimension $m \geq 2$ in the complex projective space $\mathbf{P}^{N}$.

Work of Tian and Yau [34] and Tian 33] shows that, with precisely two exceptions, every compact complex surface with positive first Chern class admits an Einstein-Kähler metric.

Remark 4.9. (Deformation of positive Einstein-Kähler manifolds) In higher dimensions, work of Nadel [25] and Siu [31] shows that if $m / 2 \leq k \leq m+1$, then the Fermat hypersurface of degree $k$ in $\mathbf{P}^{m+1}$ admits an Einstein-Kähler metric 
with positive Ricci curvature. Nadel also proved existence of positive EinsteinKähler metrics on certain branched coverings of projective space. These examples can be deformed; this is an immediate consequence of a theorem of LeBrun and Simanca [21] regarding deformations of extremal Kähler metrics. Precisely, if $\left(M, J, g_{M}\right)$ is a positive Einstein-Kähler manifold with no non-trivial holomorphic vector fields, their result implies that every sufficiently small deformation of the complex manifold $(M, J)$ also admits an Einstein-Kähler metric.

If $(M, J)$ is a smooth hypersurface of degree $k \leq m+1$ in $\mathbf{P}^{m+1}$, then every small deformation is realized by a hypersurface in the same projective space. Consequently, there is a moduli space of dimension $\left(\begin{array}{c}m+k+1 \\ k\end{array}\right)-(m+2)^{2}+1$ (the dimension of the space of monomials minus the dimension of Aut $\mathbf{P}^{m+1}$ ) consisting of Einstein-Kähler structures (complex structure and compatible Kähler metric) on the smooth manifold underlying the Fermat hypersurface. Since for $m \geq 3$ every smooth, irreducible hypersurface in $\mathbf{P}^{m+1}$ has $h^{1,1}=b^{2}=1$ (by the Lefschetz Hyperplane Theorem), every holomorphic line bundle over such a manifold admits an Hermitian structure for which the line bundle data are $\sigma$-constant.

4.3. Vector Bundles. This section describes some examples of data satisfying the hypotheses of Theorem $D$ As mentioned in the introduction, $\sigma$-constancy for a vector bundle of rank $n>1$ is a strong condition, nowhere nearly as flexible as the corresponding notion for line bundles. This is expected, since the base metric $g_{D}$ lives on a space of dimension $d$, while it is necessary to control $d+n-1$ curvature eigenvalues. The examples here are all chosen so that the exceptional divisor of the blow-up is particularly simple, either a homogeneous space, or else a product $D \times \mathbf{P}^{n-1}$. Nonetheless, there are interesting metrics, many of which seem to be new.

Homogeneous vector bundles. Let $D$ be a compact, homogeneous Kählerian manifold, and fix a maximal compact group $K \subset \operatorname{Aut}^{0}(D)$, endowed with a biinvariant measure of unit volume. The group $K$ is unique up to conjugacy in $\operatorname{Aut}^{0}(D)$. Furthermore, $K$ acts transitively on $D$, and every de Rham class (in particular, every Kähler class) contains a unique $K$-invariant representative, obtained from an arbitrary representative by averaging.

Remark 4.10. When $D$ is simply-connected, it is known that $D$ is rational, and that every holomorphic vector bundle over $D$ is homogeneous. Generally, a compact, homogeneous Kähler manifold is the Kähler product of a flat torus and a rational homogeneous space, see Matsushima 24], or the book of Besse [3] for a more detailed expository treatment.

The Ricci form of a $K$-invariant Kähler metric is $K$-invariant and represents $2 \pi c_{1}(D)$. In other words, there is only one $K$-invariant Ricci form, and its eigenvalues, with respect to a $K$-invariant Kähler form are $K$-invariant functions, i.e. constants.

Suppose $p: E \rightarrow D$ is a homogeneous holomorphic vector bundle, namely is induced by a representation of $K$ on $G L(n, \mathbf{C})$, and that the ruled manifold $M=$ $\mathbf{P}(E)$ is $K$-homogeneous (for example, if $E$ is irreducible). Then the tautological bundle $p: L \rightarrow M$ is a homogeneous line bundle, and by averaging over $K$ it is clear that for every Kähler class on $M$, there exists a Kähler form $\omega_{M}$ representing 
the chosen class, and an Hermitian structure in $L$, such that the data $p:(L, h) \rightarrow$ $\left(M, g_{M}\right)$ are $\sigma$-constant.

A tubular neighbourhood of the zero section of $L$ is obtained by blowing up a tubular neighbourhood of the zero section of $E$, so it is clear that these neighbourhoods are either both pseudoconvex or both not pseudoconvex. But in $L$, pseudoconvexity is equivalent to non-positivity of the curvature. Thus, under the assumptions of Corollary D.1, the hypotheses of Theorem D are satisfied, so the total space of $E$ (or the disk subbundle) admits complete metrics of constant scalar curvature.

Finally, consider the $K$-invariant Ricci form $\rho_{M}$ on $M$. The restriction of $\rho_{M}$ to a fibre of $\pi: M \rightarrow D$ is the Ricci form of a Fubini-Study metric, so the form $\rho_{M}-n \gamma$ vanishes on $\operatorname{ker} \pi_{*}$, hence is pulled back from $D$. Provided this form is non-positive, there is an Einstein-Kähler metric on the total space of $E$ or the disk subbundle. We shall not pursue this avenue; a detailed (partial) classification of cohomogeneity-one Einstein-Kähler metrics has been accomplished by Dancer and Wang [6], see also Podesta and Spiro [28].

Sums of line bundles. Let $E=\Lambda \otimes \mathbf{C}^{n}$ be a sum of $n$ copies of an Hermitian line bundle over a base space such that $(\Lambda, h) \rightarrow\left(D, g_{D}\right)$ is $\sigma$-constant. Then the projectivization is a product manifold $M=D \times \mathbf{P}^{n-1}$, and the tautological bundle is $\tau_{E}=\pi_{1}^{*} \Lambda \otimes \pi_{2}^{*} \mathcal{O}_{\mathbf{P}^{n-1}}(-1)$. It is clear that the induced Hermitian structure on $L=\tau_{E}$ is $\sigma$-constant with respect to the obvious metric on $M$.

As a partial complement to this remark, observe that if $L_{1}$ and $L_{2}$ are holomorphic line bundles over a compact manifold $D$, and if exactly one of $L_{1}^{*} \otimes L_{2}$ and $L_{2}^{*} \otimes L_{1}$ admits a non-trivial holomorphic section, then there do not exist metrics $g$ and $h$ such that the data $\left(L_{1} \oplus L_{2}, h\right) \rightarrow(D, g)$ are $\sigma$-constant. This generalizes the remark about Hirzebruch surfaces that was made following Theorem D As in that case, the projective bundle $M=\mathbf{P}\left(L_{1} \oplus L_{2}\right)$ has non-reductive automorphism group, hence (by a theorem of Lichnerowicz) does not admit a Kähler metric of constant scalar curvature. Considerations of this type rather seriously restrict the possibility of finding sums of line bundles to which Theorem D applies. For example, if $D=\mathbf{P}^{d}$ (or more generally, is a compact, irreducible, rank-one Hermitian symmetric space), then the only sums of line bundles satisfying the hypotheses of Theorem $\mathrm{D}$ are as in the previous paragraph.

Stable bundles over curves. Many of the observations below regarding ruled manifolds over curves were made by Fujiki in the context of seeking extremal Kähler metrics on ruled manifolds, see [9].

Let $C$ be a smooth, compact Riemann surface of genus at least two, and let $E \rightarrow C$ be a holomorphic vector bundle of rank $n$ and degree $k$. We will use a theorem of Narasimhan-Seshadri [26], as formulated by Atiyah-Bott [1, to show that when $E$ is stable there is an Hermitian metric in $E$ satisfying the hypotheses of Theorem D

Equip $C$ with the (unique up to isometry) Kähler form $\omega_{C}$ of unit area and constant Gaussian curvature, so that $c_{1}(E)=k \omega_{C}$. It is well-known (see [1], Sections 6 and 8, for example) that stable holomorphic bundles $E$ correspond to irreducible representations $\Gamma_{R} \rightarrow U(n)$, where $\Gamma_{R}$ is a central extension of the fundamental group:

$$
0 \rightarrow \mathbf{R} \rightarrow \Gamma_{R} \rightarrow \pi_{1}(C) \rightarrow 0
$$


It follows that if $E$ is stable then there is a family of metrics with constant Ricci eigenvalues on $\mathbf{P}(E)$. Indeed, because the universal cover $\Delta$ of $C$ is Stein (and contractable) the universal cover of $\mathbf{P}(E)$ may be identified holomorphically with $\Delta \times \mathbf{P}\left(\mathbf{C}^{n}\right)$. Equipping this with a product metric Poincaré $\times$ Fubini-Study, it follows that if $E$ is stable then $M=\mathbf{P}(E)$ is the quotient $\Delta \times \mathbf{P}\left(\mathbf{C}^{n}\right) / \pi_{1}(C)$ where the action is by isometries. Scaling the two factors gives a two-parameter family of Kähler forms on $M$ whose Ricci eigenvalues are constant, and whose eigenbundles are the vertical tangent bundle of $M$ and its orthogonal complement. Thus $h^{1,1}(M) \geq 2$.

The Leray-Hirsch Theorem (see for example [14], pp. 31ff.) implies that, as a module over $H^{*}(C, \mathbf{R})$, the cohomology ring $H^{*}(M, \mathbf{R})$ is generated by the first Chern class $\zeta$ of the tautological bundle $\tau_{E}$ subject to the relation

$$
0=\zeta^{n}-c_{1}(E) \zeta^{n-1}=\zeta^{n}-k \omega_{C} \zeta^{n-1} .
$$

In particular, $h^{2}(M)=2$, so by the observations made in the previous paragraph, every two-dimensional cohomology class is represented by a form whose pullback to $\Delta \times \mathbf{P}^{n-1}$ is a (possibly indefinite) linear combination of the Poincaré form and the Fubini-Study form. Kähler classes are exactly those classes whose representatives pull back to positive combinations of these metrics. Fix a Kähler class on $M$, and let $g_{M}$ be the distinguished representative. Every holomorphic line bundle $p: L \rightarrow\left(M, g_{M}\right)$ admits an Hermitian structure $h$, unique up to scaling, whose curvature form is a combination of the Poincaré and Fubini-Study forms; thus the data $p:(L, h) \rightarrow\left(M, g_{M}\right)$ are $\sigma$-constant.

Lemma 4.11. Let $E \rightarrow C$ be a holomorphic vector bundle of rank $n$ and degree $k$ over a compact Riemann surface of genus $g \geq 2$. Assume $C$ and $M=\mathbf{P}(E)$ are equipped with metrics as above, and let $\omega_{F}$ denote the push-forward to $M$ of the integral Fubini-Study form on $\Delta \times \mathbf{P}^{n-1}$. Then the curvature form $\gamma$ of the tautological bundle of $E$ and the Ricci form $\rho_{M}$ are given by

$$
\frac{1}{2 \pi} \gamma=\frac{k}{n} \omega_{C}-\omega_{F}, \quad \frac{1}{2 \pi} \rho_{M}=(2-2 g) \omega_{C}+n \omega_{F} .
$$

Proof. Write $\gamma=k_{1} \omega_{C}+k_{2} \omega_{F}$. Then $k_{2}=-1$ since the restriction of $\tau_{E}$ to a fibre is $\mathcal{O}_{\mathbf{P}^{n-1}}(-1)$. By equation (4.10) and a short calculation, $k_{1}=k / n$. To see the Ricci form is as claimed, pull back to the universal cover, where the metric is a product, and recall that $\omega_{C}$ has unit area.

If $L \rightarrow M$ is a line bundle whose first Chern class is non-positive, then the hypotheses of Theorems $\mathrm{B}$ and $\mathrm{C}$ are satisfied. Further, by Lemma 4.11 the tautological bundle $L=\tau_{E}$ has non-positive first Chern class if and only if $k=\operatorname{deg} E \leq 0$. In this event, the hypotheses of Theorem $\mathrm{D}$ are satisfied, and the total space of $E$ (or the disk subbundle) admits complete Kähler metrics of constant scalar curvature. Finally, Lemma 4.11 implies $\rho_{M}+n \gamma=2 \pi(2-2 g+k) \omega_{C}$ so by Theorem D the disk subbundle of $E$ supports a complete Einstein-Kähler metric of negative curvature.

4.4. Metrics of Finite Fibre Area. Metrics of finite fibre area arise in the momentum construction when the momentum interval is bounded. In this case the most convenient normalization is to take the momentum interval $I$ to be symmetric about $\tau=0$, rather than insisting that $\inf I=0$. The requirement that 
$\omega_{M}(\tau)=\omega_{M}-\tau \gamma$ be positive-definite for all $\tau \in I$ no longer imposes a sign restriction on $\gamma$. The main difficulty is that the problem of constructing a complete, finite-volume Kähler metric of constant scalar curvature is over-determined: There are two boundary conditions - namely $\varphi=0$, and $\left|\varphi^{\prime}\right|=0$ or 2-that must be satisfied at each end of the momentum interval, and only three parameters (the initial conditions, and the value of the scalar curvature).

Metrics on compact manifolds. Theorem 4.12 below is an existence result for Kähler metrics of constant (positive) scalar curvature on certain compact manifolds to which the momentum construction is applicable. It was proven in [12, and is included here for two purposes: To suggest the type of theorem to be expected for (non-compact) metrics of finite fibre area, and to indicate the parts of the proof that generalize with no extra effort. The important philosophical point is that on a compact manifold, it is not generally the case that every Kähler class is represented by a metric of constant scalar curvature.

Theorem 4.12. Let $\left(M, g_{M}\right)$ be a product of positive Einstein-Kähler manifolds, each having $b_{2}=1$, and let $p: L \rightarrow M$ be a holomorphic line bundle whose first Chern class is strictly indefinite. Then the completion $\widehat{L}=\mathbf{P}(L \oplus \mathbf{1})$ admits a Kähler metric of constant scalar curvature. In fact, the set of Kähler classes containing such a metric is a real-algebraic family that separates the Kähler cone.

Proof. (Sketch) The first step is to establish that under the hypotheses of Theorem 4.12 every Kähler class on $\widehat{L}$ is represented by a Kähler metric whose scalar curvature is an affine function of $\tau$. (This means the gradient of the scalar curvature is a global holomorphic vector field, hence that the metric is critical for a certain energy functional, see [5]. Such a metric is extremal (in the sense of Calabi). A Kähler class containing an extremal representative is an extremal class.)

Let $Q$ and $R$ be defined as in Section 2 and let $I=[-b, b]$. Set $\sigma(\tau)=\sigma_{0}+\sigma_{1} \tau$ and solve the boundary value problem

$$
\begin{aligned}
(\varphi Q)^{\prime \prime}(\tau) & =2 Q(\tau)\left(R(\tau)-\sigma_{0}-\sigma_{1} \tau\right) ; \\
\varphi( \pm b) & =0, \quad \varphi^{\prime}( \pm b)=\mp 2 .
\end{aligned}
$$

The values of $\sigma_{0}$ and $\sigma_{1}$ are determined uniquely by $Q, R$, and $b$, and a root counting argument shows that $(\varphi Q)^{\prime \prime}$ vanishes at most twice, so that $\varphi Q$-which is positive near the endpoints of $I$-is positive on $(-b, b)$. Thus equation (4.12) determines a momentum profile whose induced metric is extremal.

The scalar curvature of such a metric is constant exactly when $\sigma_{1}=0$. Direct calculation expresses $\sigma_{1}$ as a polynomial in the curvature and Ricci eigenvalues of the horizontal data; the "variables" are exactly the parameters controlling the Kähler class of the base metric. The top-degree coefficient changes sign as the parameters vary, so $\sigma_{1}$ changes sign on the Kähler cone, hence vanishes on a realalgebraic hypersurface that separates the cone.

There are substantial difficulties in extending Theorem 4.12 to compact manifolds when the base curvature is not positive. Perhaps the greatest, found by Tønnesen-Friedman [35], is that the set of extremal classes is not obviously the entire Kähler cone. In particular, on a ruled surface whose base has genus at least two, the set of classes for which the momentum construction yields an extremal 
metric is not generally the entire Kähler cone. This potentially complicates the final portion of the argument, since $\sigma_{1}$ may vanish for certain choices of eigenvalues, but the relevant parameters may not correspond to extremal classes.

Non-compact metrics of finite fibre area. By analogy with the compact case, it is desirable to search among a family of metrics whose scalar curvature may be non-constant. The natural extension is to the class of formally extremal metrics, by definition those whose scalar curvature is an affine function of the moment map. As before, the hope is to find, for each pair of boundary conditions, an affine function $\sigma_{0}+\sigma_{1} \tau$ such that the function $\varphi$ satisfying

$$
R(\tau)-\frac{1}{2 Q(\tau)}(\varphi Q)^{\prime \prime}(\tau)=\sigma_{0}+\sigma_{1} \tau
$$

matches the given boundary conditions and is non-negative. Matching the boundary conditions is easy linear algebra:

Lemma 4.13. Let $\left\{p:(L, h) \rightarrow\left(M, g_{M}\right),[-b, b]\right\}$ be $\sigma$-constant horizontal data for some $b>0$. For each pair of boundary values $\varphi^{\prime}( \pm b)$, there exists a unique choice of $\sigma_{0}$ and $\sigma_{1}$ such that the function $\varphi$ defined by (4.13) has the given boundary derivatives and satisfies $\varphi( \pm b)=0$.

The proof is simple linear algebra, identical to the compact case. This "boundarymatching" result is the only part of the proof of Theorem 4.12 that generalizes immediately, and the results of Tønnesen-Friedman suggest that there are genuine geometric complications in less restricted settings.

4.5. Other Constructions of Bundle-Adapted Metrics. Several authors have constructed bundle-adapted metrics from various points of view. With the exception of LeBrun, who worked over curves, the authors mentioned below have used the following curvature hypotheses, either implicitly or explicitly:

(i): The eigenvalues of the curvature endomorphism B are constant;

(ii): The eigenvalues of the Ricci endomorphism $\varrho$ are constant;

(iii): At each point of $M, \mathrm{~B}$ and $\varrho$ are simultaneously diagonalizable.

For brevity, data satisfying these conditions are said to be $\rho$-constant. Data that are $\rho$-constant are clearly $\sigma$-constant. Roughly, the distinction is between assuming an endomorphism has constant trace and assuming its eigenvalues are constant. It is clear that $\sigma$-constant data satisfying $\lambda \omega_{M}=\rho_{M}+k \gamma$ are $\rho$-constant; thus $\rho$-constancy is a natural curvature condition when searching for Einstein-Kähler metrics.

Historical survey. The list below is in approximate chronological order, but does not necessarily follow lines of development back to their earliest discernible origins. We offer our sincerest apologies to authors whose related work we may have overlooked.

Calabi 44 used distortion potential functions to construct complete EinsteinKähler metrics in line bundles over an Einstein-Kähler base, and in the cotangent bundle of $\mathbf{P}^{d}$, and used the same method (in [5]) to construct compact extremal Kähler metrics of non-constant scalar curvature.

Koiso and Sakane [16, 17] used the momentum map as a coordinate to construct compact Einstein-Kähler metrics of real cohomogeneity one. Their work followed Sakane [29], likely inspired by the work of Bérard-Bergery [2] on compact, 
non-homogeneous Einstein metrics. One salient point of their work is an explicit interpretation of vanishing of the Futaki invariant as an integrability condition for an ODE. At about the same time, Mabuchi [22] gave a more symplectic proof of existence of Einstein-Kähler metrics on the same spaces considered by Koiso and Sakane. Mabuchi also gave a satisfactory interpretation of the Futaki invariant in terms of convex geometry.

A symplectic approach was also taken by LeBrun [19], and his formalism was generalized by Pedersen and Poon [27. LeBrun assumed that the base $M$ was a curve, but allowed the profile to be a general (positive) function on $I \times M$. (Correspondingly the dependence upon $\tau$ of $\omega_{M}(\tau)$ is not necessarily affine, and $d \omega_{\varphi}=0$ is an additional condition.) He showed that every $S^{1}$-invariant scalar-flat Kähler metric in complex dimension 2 is locally described by a pair of functions $u$ and $w$ on $I \times M$ satisfying certain partial differential equations. His $w$ is essentially $1 / \varphi$. Remarkably, these equations are tractable when $\operatorname{dim} M=1$ and lead, for example, to the construction of scalar-flat Kähler metrics on (certain) blow-ups of ruled surfaces.

Pedersen and Poon allowed $\operatorname{dim} M>1$ and worked with torus bundles, but made assumptions that reduce their very complicated system of equations to the ODEs studied in this paper. (In most of their examples, $M$ is Einstein-Kähler and $L$ is a (possibly fractional) power of the canonical bundle.) Their examples include metrics of constant scalar curvature on line and disk bundles over projective spaces. In particular they showed that there exist Kähler metrics of zero scalar curvature on the total space of $\mathcal{O}(-k) \rightarrow \mathbf{P}^{m}$ provided that $k \geq m$. This restriction on $k$ is unnecessary, as shown by Simanca [30, also using the Calabi ansatz.

The methods of Koiso and Sakane were used in [12], and independently by Guan [10], to extend Calabi's families of extremal metrics. Hwang ([12], p. 564) mis-attributed the construction to Koiso and Sakane, overlooking the fact that Calabi ([4], p. 281, equation (4.9), for example) had written the moment map and distance function in the manner of equation (2.2) above. However, Calabi seems to have made the observation in passing, and did not emphasize the use of momentum coordinates.

Koiso [15] and Guan [11] used the method of Koiso and Sakane to study Hamilton's Ricci flow for Kähler metrics and how it may fail to converge; the concept of a quasi-Einstein Kähler metric is introduced in the latter two papers. A quasiEinstein Kähler metric is the Kählerian analogue of a Ricci soliton, introduced by R. Hamilton.

Dancer and Wang [6] and Podesta and Spiro 28] independently used the techniques of Koiso and Sakane to obtain a partial classification of Einstein-Kähler metrics having real hypersurface orbits under the action of the isometry group.

Engman [7, 8] and Taimanov 32] used the momentum construction (sometimes implicitly) to study spectral geometry of surfaces of revolution. Momentum coordinates are ideal, since the Laplacian reduces to a second-order, linear, ordinary differential operator.

Tønnesen-Friedman [35] used the Calabi ansatz to study existence of extremal Kähler metrics on some ruled surfaces. Some of her examples are of geometric interest for the following reason. If $(M, J)$ is a compact complex manifold that admits an extremal Kähler metric in some Kähler class, then there are two properties that might generally be hoped for: 
- Every Kähler class contains an extremal representative;

- Extremal metrics in a fixed class on $M$ are unique up to the action of the automorphism group.

Tønnesen-Friedman found families of complex surfaces (admitting extremal metrics) for which at least one of the preceding statements fails.

The number of geometric, analytic, and physical applications of the momentum construction (in addition to its independent discovery by workers in different fields) indicates its potential interest to the mathematical community. It is our hope that the expository elements of this account will bring the technique to a wider audience, and that many will find bundle-adapted metrics a useful testing ground for a variety of investigations.

\section{REFERENCES}

[1] M. F. Atiyah and R. Bott: The Yang-Mills equations over Riemann surfaces, Phil. Trans. Royal Soc. London A, 308 (1982), 524-615. MR 85k:14006

[2] L. Bérard-Bergery: Sur de nouvelles variétés Riemannian d'Einstein, Pub. de l'Institute E. Cartan, Nancy, 4 (1982), 1-60. MR 85b:53048

[3] A. L. Besse: Einstein manifolds, Ergebnisse der Mathematik und ihrer Grenzgebiete; 3 Folge, Band 10, Springer-Verlag, Berlin, New York, 1987. MR 88f:53087

[4] E. Calabi: Métriques Kähleriennes et fibrés holomorphes, Annales Scientifiques de l'École Normale Supérieure 12 (1979), 268-294. MR 83m:32033

[5] E. Calabi: Extremal Kähler metrics, in Seminars on differential geometry (S. T. Yau, ed.), Annals of Math. Studies, Princeton Univ. Press, 1982, 259-290. MR 83i:53088

[6] A. Dancer and M. Y. Wang: Kähler-Einstein metrics of cohomogeneity one, and a bundle construction for Einstein-Hermitian metrics, preprint, 1997.

[7] M. Engman: New spectral characterization theorems for $S^{2}$, Pacific J. Math., 154 (1992), 215-229. MR 93d:58173

[8] M. Engman: Trace formula for $S^{1}$ invariant Green's operators on $S^{2}$, Manuscripta Math., 93 (1997), 357-368. MR 99c:58160

[9] A. Fujiki: Remarks on extremal Kähler metrics on ruled manifolds, Nagoya Math. J., 126 (1992), 89-101. MR 94c:58033

[10] Z.-D. Guan: Existence of extremal metrics on compact almost homogeneous Kähler manifolds with two ends, Trans. AMS 347 (1995), no. 6, 2255-2262. MR 96a:58059

[11] Z.-D. Guan: Quasi-Einstein metrics, Int. J. Math 6 (1995) no. 3, 371-379. MR 96e:53060

[12] A. D. Hwang: On existence of Kähler metrics with constant scalar curvature, Osaka J. Math., 31 (1994), 561-595. MR 96a:53061

[13] S. Kobayashi: Transformation groups in differential geometry, Ergebnisse der Mathematik und ihrer Grenzgebiete; Band 70, Springer-Verlag, Berlin, New York, 1972. MR 50:8360

[14] S. Kobayashi: Differential geometry of complex vector bundles, Publications Nihon Sugakki 15, Iwanami Shoten (Tokyo), Princeton University Press (Princeton), 1987. MR 89e:53100

[15] N. Koiso: On rotationally symmetric Hamilton's equation for Kähler-Einstein metrics, in Recent topics in differential and analytic geometry, Advanced Studies in Pure Math 18-I, 1990, 327-337. MR 93d:53057

[16] N. Koiso and Y. Sakane: Non-homogeneous Kähler-Einstein metrics on compact complex manifolds in Curvature and topology of Riemannian manifolds, Springer Lecture Notes in Mathematics 1201, 1986, 165-179. MR 88c:53047

[17] N. Koiso and Y. Sakane: Non-homogeneous Kähler-Einstein metrics on compact complex manifolds II, Osaka J. Math., 25 (1988), 933-959. MR 90e:53061

[18] C. LeBrun: Counter-Examples to the Generalized Positive Action Conjecture, Commun. Math. Phys. 118 (1988) 591-596. MR 89f:53107

[19] C. Lebrun: Explicit self-dual metrics on $\mathbf{C} P_{2} \sharp \ldots \mathbf{C} P_{2}$, J. Diff. Geom., 34 (1991) 223-253. MR 92g:53040

[20] C. Lebrun: Scalar-flat Kähler metrics on blown-up ruled surfaces, J. Reine Angew. Math., 420 (1991), 161-177. MR 92i:53066 
[21] C. Lebrun and S. R. Simanca: Extremal Kähler metrics and complex deformation theory, Geometric and Functional Analysis, 4 (1994), 298-336. MR 95k:58041

[22] T. Mabuchi: Einstein-Kähler forms, Futaki invariants, and convex geometry on toric Fano varieties, Osaka J. Math, 24 (1987), 705-737. MR 89c:53074

[23] Y. Matsushima: Remarks on Kähler-Einstein manifolds, Nagoya Math. J., 46 (1972), 161-173. MR 46:2615

[24] Y. Matsushima: Sur les espaces homogènes kählériens d'un groupe de Lie réductif, Nagoya Math. J., 11 (1957), 53-60. MR 19:315c

[25] A. M. Nadel: Multiplier ideal sheaves and Kähler-Einstein metrics of positive scalar curvature, Annals of Math., 132 (1990), 549-596. MR 92d:32038

[26] M. S. Narasimhan and C. S. Seshadri: Stable and unitary vector bundles on compact Riemann surfaces, Annals of Math. 82 (1965), 540-567. MR 32:1725

[27] H. Pedersen and Y.-S. Poon: Hamiltonian construction of Kähler-Einstein metrics and Kähler metrics of constant scalar curvature, Comm. Math. Phys. 136 (1991), 309-326. MR 92b:53073

[28] F. Podesta and A. Spiro: Kähler manifolds with large isometry group, Osaka J. Math. 36 (1999), 805-833. MR 2001h:53062

[29] Y. Sakane: Examples of Einstein-Kähler manifolds with positive Ricci tensor, Osaka J. Math. 23 (1986), 585-616. MR 87k:53111

[30] S. R. Simanca: Kähler metrics of constant scalar curvature on bundles over $\boldsymbol{C P}_{n-1}$, Math. Ann. 291 (1991), 239-246. MR 92m:53115

[31] Y.-T. Siu: The existence of Kähler-Einstein metrics on manifolds with positive anticanonical bundle and suitable finite symmetry group, Annals of Math. 127 (1988), 585-627. MR 89e:58032

[32] I. A. Taimanov: Surfaces of revolution in terms of solitons, Ann. Global Analysis and Geom., 15, No. 5, Oct. 1997, 419-435. MR 99f:53005

[33] G. Tian: On Calabi's conjecture for complex surfaces with positive first Chern class, Invent. Math. 101 (1990), 101-172. MR 91d:32042

[34] G. Tian and S.-T. Yau: Existence of Kähler-Einstein metrics on complete Kähler manifolds and their applications to algebraic geometry, in Mathematical Aspects of String Theory, San Diego, California, S.-T. Yau ed., World Scientific, 1987, pp. 574-628. MR 89m:81001

[35] C. Tønnesen-Friedman: Extremal Kähler metrics on ruled surfaces, Odense Univ. Preprint No. 36, 1997.

Department of Mathematics and Computer Science, College of The Holy Cross, Worcester, MassachusetTs 01610-2395

E-mail address: ahwang@mathcs.holycross.edu

Department of Mathematics and Statistics, University of Edinburgh, Edinburgh, EH9 3JZ, UK

E-mail address: michael@maths.ed.ac.uk 